\title{
Phonon Transport within Periodic Porous Structures - From Classical Phonon Size Effects to Wave Effects
}

\author{
Yue Xiao, Qiyu Chen ${ }^{1}$, Dengke $\mathrm{Ma}^{2,3}$, Nuo Yang ${ }^{2,4 *}$ and Qing Hao ${ }^{1 *}$
}

Tailoring thermal properties with nanostructured materials can be of vital importance for many applications. Generally classical phonon size effects are employed to reduce the thermal conductivity, where strong phonon scattering by nanostructured interfaces or boundaries can dramatically suppress the heat conduction. When these boundaries or interfaces are arranged in a periodic pattern, coherent phonons may have interference and modify the phonon dispersion, leading to dramatically reduced thermal conductivity. Such coherent phonon transport has been widely studied for superlattice films and recently emphasized for periodic nanoporous patterns. Although the wave effects have been proposed for reducing the thermal conductivity, more recent experimental evidence shows that such effects can only be critical at an ultralow temperature, i.e., around $10 \mathrm{~K}$ or below. At room temperature, the impacted phonons are mostly restricted to hypersonic modes that contribute little to the thermal conductivity. In this review, the theoretical and experimental studies of periodic porous structures are summarized and compared. The general applications of periodic nanostructured materials are further discussed.

Keywords: Phononic crystal; Classical phonon size effect; Coherence; Nanopore

Received 15 May 2019, Accepted 9 September 2019

DOI: $10.30919 /$ esmm5f237

\section{Introduction - Challenges in manipulating heat as waves}

Nanostructured materials introduce unique opportunities to tailor the intrinsic transport properties. In structures such as solid thin films, the reduced lattice thermal conductivity $\left(k_{L}\right)$ is often attributed to the boundary scattering of particle-like phonons, as the classical phonon size effect. When periodic nano-patterns are introduced within a structure, unique opportunities also exist in using the wave nature of lattice vibrations to modify the phonon dispersion and thus the $k_{L}$. The overall thermal conductivity $k$, consisting of both $k_{L}$ and the electronic contribution $k_{E}$, can also be largely suppressed. This can benefit applications requiring a low $k$, such as thermoelectric energy conversion and thermal insulation materials. ${ }^{1}$ As one important research direction of reducing the thermal transport, periodic nanoporous thin films ${ }^{2-18}$ (Fig. 1a) and graphene ${ }^{19,20}$ (also known as graphene antidot lattices or GALs, ${ }^{21}$

\footnotetext{
'Department of Aerospace and Mechanical Engineering, University of Arizona, Tucson, AZ 85721 U.S.A

State Key Laboratory of Coal Combustion, Huazhong University of Science and Technology (HUST), Wuhan 430074, China

${ }^{3}$ NNU-SULI Thermal Energy Research Center (NSTER) \& Center for Quantum Transport and Thermal Energy Science (CQTES), School of Physics and Technology, Nanjing Normal University, Nanjing 210023, China

${ }^{4}$ Nano Interface Center for Energy(NICE), School of Energy and Power Engineering, Huazhong University of Science and Technology (HUST), Wuhan 430074, China

*E-mail: qinghao@email.arizona.edu; nuo@hust.edu.cn
}

as shown in Fig. 1b) have been widely studied.

In above mentioned 2D periodic porous structures, possibly coherent interference between lattice vibration waves can lead to opened phononic bandgaps. The term "coherence" is generally known for optics $^{22}$ and acoustics. ${ }^{23}$ For lattice vibration waves within a periodic nanostructured material, the forward and reflected waves by periodic interfaces or boundaries can interference to change the phonon behavior. This phenomenon happens when the phonon phase is preserved during their transport processes. With coherent phonon transport, the modified phonon density of states (DOS) and phonon group velocities can largely reduce $k_{L}$. When the phonon wave effects are negligible, classical size effects of particle-like incoherent phonons should be considered. In analogy to photonic crystals with periodic cavities to manipulate light, ${ }^{24,25}$ periodic nanoporous films and antidot lattices are referred to as "phononic crystals." Such phononic effects has been intensively studied for superlattice thin films with atomically smooth interfaces between alternating layers, in which coherent phonon transport becomes dominant for $<5 \mathrm{~nm}$ periods at $300 \mathrm{~K}^{26,27}$ In comparison, nanopores within thin films or GALs may play the same role as interfaces within superlattices. Their periodicity modifies phonon dispersions, whereas their rough edges act as defects to scatter phonons.

In general, it is more challenging to observe room-temperature phononic effects in nanoporous films due to the technical difficulty in achieving: 1) ultrafine patterns with sub-10 $\mathrm{nm}$ periodic length or pitch $p$; and 2) smooth pore edges with minimized defects. Compared with photons, phonons with much shorter wavelengths are more difficult to be manipulated for their wave effects using periodic nanostructures. This article reviews existing studies on atomic to nanoscale periodic porous structures to tune the phonon transport. The limitation and important applications of phononic effects are discussed. The discussions cover two-dimensional (2D) periodic porous thin films and 
GALs, and three-dimensional (3D) porous nano-cages (Figs. 1a-c).

\section{Requirements to achieve strong phononic effects with periodic porous materials}

As an overview, the general guidance to achieve remarkable phononic effects is given below. Following the studies on superlattices, a sub-10 $\mathrm{nm}$ pitch is necessary for periodic nanoporous materials to match the short wavelength of majority phonons at room temperature $(1-10 \mathrm{~nm}$ at $300 \mathrm{~K}$ for bulk $\mathrm{Si}^{28,}{ }^{29}$ ) because the impacted phonons should have wavelengths comparable to or shorter than the periodic length of the structure. Similar to the Bragg gaps for photonic crystals, the frequency of phonons impacted by the periodic structure can be approximated as $f$ $=\pi v_{\text {avg }} / p$, in which $v_{\text {avg }}$ is the averaged sound velocity. For $p=100 \mathrm{~nm}$, phonons with $f \sim 200 \mathrm{GHz}$ or below can be impacted, instead of majority heat-carrying phonons at a few $\mathrm{THz}$ in bulk Si. The overall impact on $k_{L}$ is very limited. In the literature, the smallest $p$ is $34 \mathrm{~nm}$ for measured nanoporous Si films ${ }^{14}$ (see Table 1). Further reducing this $p$ value to $10 \mathrm{~nm}$ or below is limited by the $\sim 5 \mathrm{~nm}$ spatial resolution of the state-of-the-art electron beam lithography (EBL). For a film with its thickness $t$, the smallest pore diameter $d$ is also restricted by the aspect ratio $t / d<3$ for dry etching, i.e., $d>t / 3$. ${ }^{10}$ For atomic-thick materials, the limitation due to the aspect ratio is removed but accurate thermal measurements still remain as a challenge.

Compared to smooth interfaces within a superlattice thin film, pore edges within nanoporous structures should be smooth enough to keep the phase information of coherent phonons before and after the pore-edge scattering. Phononic effects require specular phonon reflection on all boundaries because diffuse phonon scattering will destroy the coherent phonon phase. ${ }^{30}$ In Ziman's theory, the probability $P(\lambda)$ for specular reflection can be estimated as $P(\lambda)=\exp \left[-16 \pi^{2}(\delta / \lambda)^{2}\right]$, in which $\delta$ is the average boundary roughness and $\lambda$ is phonon wavelength. ${ }^{31,32}$ Following this $P(\lambda)$ expression, even $\delta$ as small as $1 \mathrm{~nm}$ can yield completely diffusive phonon scattering to destroy phononic effects. In real samples, a layer of 1-2 nm native oxide on the pore sidewalls is revealed by high-resolution transmission electron microscopy (TEM). ${ }^{33}$ Such amorphous pore edges are inevitable due to the structure damage by the pore-drilling process and strong oxidation of nanostructured surfaces. Further considering the diffusive phonon refection on the top and bottom surfaces of a thin film, strong phonon coherence at $300 \mathrm{~K}$ is not anticipated for reported periodic nanoporous $\mathrm{Si}$ thin films. In this aspect, the claimed room-temperature phononic effects for pores with $2.5 \mathrm{~nm}$ surface roughness ${ }^{2}$ may be attributed to measurement errors using a microdevice.

In principle, coherent phonon transport also requires structure sizes to be much smaller than majority phonon mean free paths (MFPs) because the internal phonon scattering inside material can also destroy the coherent phonon phase. ${ }^{34}$ This issue is less critical because significant percentage of heat is still carried by phonons with very long MFPs. At $300 \mathrm{~K}$, first-principles calculations ${ }^{28}$ suggest that $50 \%$ of the room-temperature $k_{L}$ is contributed by phonons with MFPs longer than 1 $\mu \mathrm{m}$. Along this line, the phonon MFP comparison with $p$ has also been proposed to justify the importance of phononic effects. ${ }^{2,4}$ However, this argument is inconsistent with the understanding from comparable photonic crystals, where the wavelength comparison with $p$ is always used to justify the wave effect.

Currently, there still exist some knowledge gaps between theoretical and experimental studies. In real samples, smooth pore edges and a sub-10 nm pitch are hard to be achieved. However, such "perfect" structures are widely studied with molecular dynamics (MD) simulations and strong wave effects are often suggested. ${ }^{35-40}$ In a few MD simulations, pore-edge defects ${ }^{41}$ and structure disorder ${ }^{42}$ are further considered to check their impact on phononic effects. In this review, incoherent phonon transport is mainly discussed for nanoporous films, whereas strong coherent transport is suggested by MD simulations for ideal porous structures. Experimentally, experimental validation is still lacking for the simulated 3D nanoporous structures in Section 6 and wave effects can often be overestimated in MD simulations.

\section{Theoretical and experimental methods used for nanoporous films}

As an overview, the existing theoretical and experimental methods used for the thermal studies of nanoporous materials are summarized here.

For thermal conductivity calculations, $k_{L}$ predictions are often based on the Boltzmann transport equation (BTE) assuming incoherent phonon transport. Pore edges are assumed to diffusively reflect all incident phonons. The wave effects are neglected in this situation. Due to the challenge in numerically solving the phonon BTE for complicated structure geometry and frequency-dependent phonon MFPs, phonon Monte Carlo (MC) simulations are often employed as an alternative way to solve the BTE. ${ }^{43-45}$ In these simulations, transport of individual phonons across one single period as the computational domain is tracked, using a boundary condition assuming a periodic heat flux with a constant virtual wall temperature for periodic structures. When the simulation converges, $k_{L}$ can be computed with the heat flow and temperature difference across the computational domain.

Without computing the exact temperature profile, various analytical models are also proposed, with a focus on how the bulk phonon MFP is modified by the nanoporosity. The lattice thermal conductivity $k_{L}$ can be calculated by replacing the bulk phonon MFP with the effective phonon MFP within the nanoporous structure. The (a)

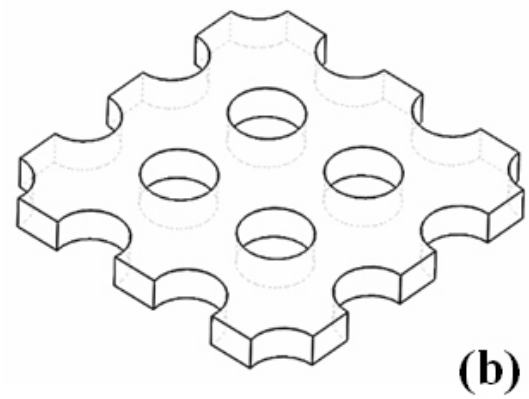

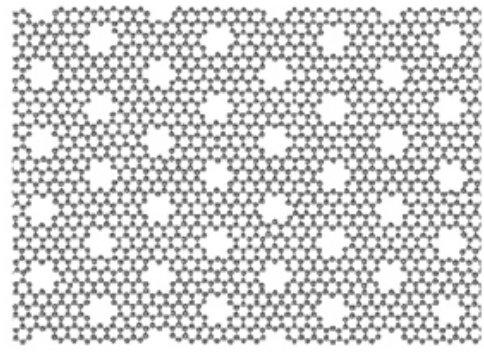

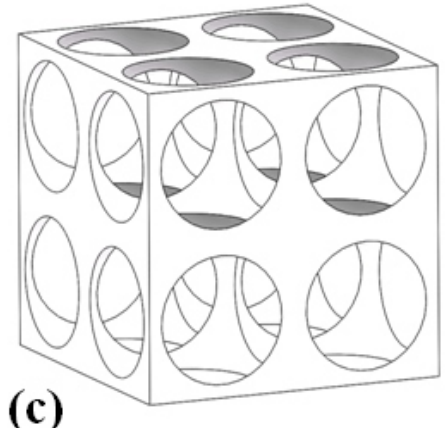

(c)

Fig. 1 The structure of (a) a periodic nanoporous film, (b) a hexagonal GAL, and (c) a spherical porous 3D phononic crystal. 
efforts of analytical modeling are reviewed in Section 4.1.

For ultrafine nanoporous patterns, possible phononic effects can be revealed by MD simulations that track the trajectories of individual atoms interacting with other atoms. The structure sizes are usually limited to $\sim 10,000$ atoms in these atomistic simulations. In practice, MD simulations are inapplicable to the measured nanoporous Si films with a minimum pitch as $34 \mathrm{~nm} .{ }^{14}$ To incorporate possible phononic effects into the phonon studies of these large structures, other simplified approaches are proposed for general periodic nanostructures, which can be found in Section 7.

In structures like GALs, the atomic Green's function (AGF) method may also be used to compute the phonon transmission across the structure. ${ }^{46}$ The phonon thermal conductance can be derived under the Landauer formalism. Again this calculation is restricted to small computational domains, instead of structures with a pitch of tens of nanometers. The general problem for the AGF method lies in that the anharmonic effect for phonon-phonon scattering cannot be easily incorporated.

In thermal measurements, three major techniques are used: (1) measurements using a suspended microdevice, (2) micro time-domain thermoreflectance ( $\mu$-TDTR) measurements, and (3) two-laser Raman thermometry. The microdevice measurements can be further divided into two approaches. In one approach, the measured sample is transferred onto a microdevice, in which the sample bridges two suspended islands with patterned metal-line heaters/thermometers., ${ }^{2,}, 33$ The thermal conductivity of the sample can be computed based on the measured steady-state temperature difference and heat flow along the sample when one island is heated up with a DC current. In a different approach, the device layer of a silicon-on-insulator (SOI) wafer is fabricated into a microdevice with the measured part patterned with periodic pores. ${ }^{8,47-50}$ The measurements are still performed with electrical heating and temperature sensing using deposited metal lines or films. Such a setup eliminates the sample-device thermal contact issue and possible sample damage during its transfer process. On the other hand, the $\mu$-TDTR measurements ${ }^{3,11,12,51-54}$ and two-laser Raman thermometry ${ }^{13,55}$ are both contactless measurement techniques. Again a suspended device is fabricated from the device layer of a SOI wafer and is patterned with nanopores. In the $\mu$-TDTR technique, the suspended device consists of an Al-coated pad in the middle and the measured nanoporous films on both sides of the $\mathrm{Al}$ pad. In measurements, this $\mathrm{Al}$ pad is heated by a pulsed laser beam and the $\mathrm{Al}$ pad is then cooled down by heat conduction through the nanoporous films. The temporal temperature evolution of the $\mathrm{Al}$ pad is monitored using a continuous-wave laser beam. The in-plane thermal conductivity of the nanoporous film can then be extracted from the thermal analysis of this transient measurement. In the two-laser Raman thermometry measurements, ${ }^{13,55}$ a spatially fixed laser generates a localized steady-state heating, whereas a low power probe laser measures the spatially-resolved temperature profile based on the temperature-dependent Raman frequency of the optical phonons in the material. The in-plane thermal conductivity can then be extracted from the measured 2D temperature profile.

The cross-plane thermal conductivity of a nanoporous film can be measured by the standard TDTR technique, with Al coating across the whole film as the optical transducer., ${ }^{7,56}$ A brief discussion can be found in Section 4.4.

\section{Periodic nanoporous films (quasi-2D phononic crystal)}

For widely studied periodic nanoporous Si films, phononic effects are negligible above room temperature in measured samples because the diffusive pore-edge phonon scattering can destroy the coherent phonon phase and large feature sizes have limited impact on majority heatcarrying phonons. In existing studies, phononic effects can only be confirmed at cryogenic temperatures, where the dominant phonon wavelengths scale up with $1 / T$, with $T$ as the absolute temperature. ${ }^{57}$ Such trends are consistent with recent measurements with phononic effects determined below $14 \mathrm{~K}$ for Si films with $p>100 \mathrm{~nm}$, or below $10 \mathrm{~K}$ for $p=300 \mathrm{~nm}^{52}$ In nanoporous $\mathrm{SiN}_{\mathrm{x}}$ films, strong phononic effects have been observed at sub-Kelvin temperatures for a pitches $p$ of 970 and $2425 \mathrm{~nm}^{18}$

In most cases, the classical phonon size effects are still the major mechanism for the thermal conductivity reduction and are the focus of the analytical modeling given below. The experimental studies are summarized for periodic nanoporous thin films, with a focus on how to justify the phononic effects. Most discussions are based on the in-plane $k_{L}$ of nanoporous Si thin films. A few studies can be found for anisotropic $k_{L}$ calculations $^{58,59}$ and cross-plane $k$ measurements. ${ }^{7,56}$

\subsection{Modeling the in-plane $k_{L}$ of periodic nanoporous thin films}

For nanoporous thin films with incoherent phonon transport, accurate in-plane $k_{L}$ can be given by phonon MC simulations. ${ }^{43-45}$ Other than complicated phonon MC simulations considering frequency-dependent phonon MFPs, analytical modelling has also be carried out using an effective phonon $\operatorname{MFP}\left(\Lambda_{e f f}\right)$ in the kinetic relationship:

$$
k_{L}=F(\Phi) C v_{g} \Lambda_{e f f} / 3
$$

Here the phonon specific heat $C$ and phonon group velocity $v_{g}$ are unchanged from the bulk values. The additional factor $F(\Phi)$ accounts for the heat transfer reduction due to the porosity $\Phi$, whereas $\Lambda_{e f f}$ further addresses the phonon size effects. In many studies, $k_{L} / F(\Phi)$ is compared to that of the starting solid thin film to show the phonon size effects due to introduced nanoporosity. In accurate analysis, $k_{L}$ is integrated over the whole phonon spectrum and summed over different phonon branches. The complicated full phonon dispersion can also be considered. $^{29}$

\subsubsection{Correction factor $\boldsymbol{F}(\Phi)$}

In principle, $F(\Phi)$ can be determined by the Fourier's law analysis, e.g., as the ratio between the thermal conductances of a porous film and its nonporous counterpart. ${ }^{29,45}$ Analytical expressions of $F(\Phi)$ are also available. In early studies, the Eucken's factor is used and is given as

$$
F(\Phi)=\frac{1-\Phi}{1+\Phi / 2}
$$

which was derived for a bulk material with cubically aligned spherical pores. ${ }^{60}$ For nanoporous films, however, the Hashin-Shtrikman factor ${ }^{61}$ is found to be more accurate. This factor is expressed as

$$
F(\Phi)=\frac{1-\Phi}{1+\Phi}
$$

In fact, Eq. (3) can also be found as a special case for a 2D composite, where pores corresponds to circular inclusions with $k=0 .{ }^{62}$ The validation of Eq. (3) with the Fourier's law analysis can be found in some studies. ${ }^{29,45}$

\subsection{2 $\Lambda_{\text {eff }}$ calculations using an analytical characteristic length $L$}

As another important aspect for the analytical modelling, the effective phonon MFP $\Lambda_{e f f}$ can be computed with a MC technique based on raytracing or path sampling, ${ }^{49,63-65}$ or solving a MFP-dependent phonon 
BTE. ${ }^{66,67}$ Considering phonon scattering at pore edges and film surfaces, these techniques should be applied to individual bulk phonon MFP $\left(\Lambda_{\text {Bulk }}\right)$ to find its corresponding $\Lambda_{\text {eff }}$. This can be extremely expensive for calculations, considering the wide $\Lambda_{\text {Bulk }}$ distribution in materials like $\mathrm{Si}^{28}$ To simplify, $\Lambda_{e f f}$ is also modified from $\Lambda_{B u l k}$ based on Matthiessen's rule, i.e., $\Lambda_{e f f}=\left(1 / \Lambda_{B u l k}+1 / L\right)^{-1}$., 44, 45, 59, 68 Here a characteristic length $L$ of the nanoporous structure is introduced. A similar treatment can be found for nanowires, where the nanowire diameter is simply the characteristic length to account for the completely diffusive phonon boundary scattering. ${ }^{69}$

For periodic nanoporous films, there are mainly three key geometry parameters: pore pitch $p$ as the averaged center-to-center distance between adjacent pores, film thickness $t$ and pore diameter $d$. In typical patterns, pores are distributed on a square lattice or a hexagonal lattice. For ultrafine nanoporous patterns, the impact of the film thickness can be negligible. Only considering $d$ and $p$, various characteristic lengths have been proposed for periodic nanoporous $\mathrm{Si}$ films. Table 1 lists $L$ expressions proposed in different studies. Among these expressions, the neck width $L=p-d$ can be used to predict the lower bound of $k_{L}$. This $L$ is not expected to be accurate for pores on a square pattern because the second-nearest-neighbor pores have an expanded neck width as $L=\sqrt{2} p-d$ from $L=p-d$ for nearestneighbor pores. ${ }^{70}$ At the limit $p \rightarrow d$, however, experimental studies by Anufriev et $a l^{51}$ and Yanagisawa et al. ${ }^{3}$ suggested that $L=p-d$ became more accurate and the surface roughness was also critical. Calculations by $\mathrm{Yu}$ et $a l^{71}$ also indicated the importance of the neck width. In the reviews by Marconnet et al. ${ }^{10}$ and Nomura et al., ${ }^{72}$ the measured $k$ values in existing studies are plotted as a function of $L=p$ $-d$ for comparison purposes.

At the ballistic limit, it has been found that the geometric mean beam length (MBL) for optically thin media in radiation ${ }^{44,45}$ is simply the accurate characteristic length. As first formulated by Hottel, the MBL is the length scale for the radiative heat transfer between an isothermal gas volume and its boundary. ${ }^{73}$ In practice, the MBL is identical with the results provided by MC ray tracing (MCRT), which is used by Lacroix et $a l .^{74}$ to determine the characteristic length of a periodic nanoporous structure. In MCRT simulations, the average free traveling distance of particles between their collisions with the pore edges is statistically obtained as the characteristic length of the structure. Different from complicated MCRT simulations, MBL calculations are very simple and can be extended to arbitrary structures. For circular through-film pores, the MBL can be computed $\mathrm{as}^{56,68}$

$$
L=\frac{4 V_{\text {Solid }}}{A}=\frac{4 V(1-\Phi)}{A}=\left\{\begin{array}{cc}
\frac{4 p^{2}-\pi d^{2}}{\pi d} & \text { (square lattice) } \\
\frac{2 \sqrt{3} p^{2}-\pi d^{2}}{\pi d} & \text { (hexagonal lattice) }
\end{array},\right.
$$

where the solid-region volume $V_{\text {Solid }}$ and pore surface area $A$ are evaluated within a period. For a through-film pore, $A$ is simply $\pi d t$ as the sidewall surface area of a pore. Here $V_{\text {Solid }}$ is diverged from the volume $V$ by a factor of $(1-\Phi)$, with $\Phi$ as the porosity of the structure.

The characteristic length $L$ has also been derived for 2D porous films by Huang et al. $^{59}$ and 3D particle-in-a-host composites by Minnich and Chen. ${ }^{7}$ In principle, nanoporous materials are viewed as a special case for a particle-in-a-host composite, with zero heat conduction inside the embedded nanoparticles. In a nanocomposite, $A$ becomes the interface area between a particle and the host and $\Phi$ becomes the volumetric percentage of nanoparticles. These two studies ${ }^{59,77}$ both follow the MFP calculations of gas molecules. ${ }^{78}$ For the host region of a 3D particle-in-ahost composite or the solid region of a $2 \mathrm{D}$ porous film, the derived $L$ is proportional to $V / A$, which is in contrast with $L \sim V(1-\Phi)$ in Eq. (4). This additional $(1-\Phi)$ factor has been proposed by Machrafi and Lebon $^{79}$ to better explain the trend of $L \rightarrow 0$ at $\Phi \rightarrow 1$. In the literature, the volumetric surface or interface area $A / V$ is acknowledged as one key parameter for $k_{L}$ reduction. ${ }^{77,80}$ However, the examination here suggests $A / V(1-\Phi)$ or the MBL as a more accurate parameter for $k_{L}$ modelling. In general, analysis based on the surface-to-volume ratio $A / V$ tends to overpredict $k_{L}$ but this overprediction can be reduced using the MBL. ${ }^{44,45}$

\subsubsection{Effective characteristic length $\boldsymbol{L}_{\text {eff }}$}

In practice, none of the characteristic lengths listed in Table 1 can be accurate across the whole phonon MFP spectrum. An effective characteristic length $L_{e f f}$ is often used to match $k_{L}$ predicted by Eq. (1) and $k_{L}$ yielded by the phonon BTE. Here $\Lambda_{e f f}=\left(1 / \Lambda_{\text {Bulk }}+1 / L_{e f f}\right)^{-1}$ is used in Eq. (1) and $L_{e f f}$ is fitted. Although the MBL or $L$ given by MCRT is accurate for the ballistic regime, the divergence between $L_{e f f}$ and MBL is expected because it is not accurate to use the Matthiessen's rule to combine the boundary phonon scattering on pore edges and internal phonon scattering inside the volume. ${ }^{78}$ For circular or square pores on a square lattice, Fig. 2 compares the MBL and effective characteristic length $L_{\text {eff }}$ for nanoporous Si films. The phonon BTE solution is given by frequency-dependent phonon MC simulations. ${ }^{45}$ Similar $L_{e f f}$ values have also been extracted in a separated study. ${ }^{58}$

In Fig. 2, both MBL and $L_{e f f}$ are divided by the pitch $p$ to get a dimensionless $\Lambda_{\text {Pore }}{ }^{*}$. As a factor only depending on the geometry, $\Lambda_{\text {Pore }}{ }^{*}$ $=M B L / p$ is only a function of the porosity. Due to its dependence on the actual phonon MFP distribution relative to the structure size, $\Lambda_{\text {Pore }}{ }^{*}=$ $L_{e f f} / p$ varies slightly for $p=50,200$ and $500 \mathrm{~nm}$. The plotted $\Lambda_{\text {Pore }}{ }^{*}=L_{e f f}$ $/ p$ is averaged over these three $p$ values. In Fig. $3, k_{L}$ for $2 \mathrm{D}$ nanoporous $\mathrm{Si}$ thin films with smooth top/bottom surfaces is predicted using the MBL or $L_{\text {eff }}$ to modify $\Lambda_{\text {Bulk }}$ and thus obtain $\Lambda_{e f f}$ in Eq. (1). It can be observed that the MBL typically leads to an overpredicted $k_{L}$, whereas the extracted $L_{\text {eff }}$ can give accurate results for $p$ from 50 to $500 \mathrm{~nm}$.

Table 1 Characteristic length $L$ of periodic nanoporous Si films. Here $V$ is the volume of one period, including the pore volume. For the pore within this period, $\mathrm{A}=(\pi d) t$ is its sidewall surface area.

\begin{tabular}{lcl}
\hline Article & $L$ expression & Physical meaning \\
\hline Hopkins et al..$^{75,76}$ & $p-d$ & Neck width between adjacent pores \\
Alaie et al. & $\sqrt{p^{2}-\pi d^{2} / 4}$ & The square root of the solid area within a period \\
Hao et al. ${ }^{45}$ & $4 V(1-\Phi) / A$ & Geometric MBL \\
Huang et al. & $d / 8 \Phi$ & $\begin{array}{l}\text { Traveling distance of a particle to encounter a pore within a swept } \\
\text { volume with } t \times d \text { cross-section area }\end{array}$ \\
\hline
\end{tabular}




\subsubsection{Influence of the film thickness}

In addition to the characteristic length of a $2 \mathrm{D}$ periodic porous pattern, the film thickness $t$ can further influence the in-plane $k_{L}$ by diffusive film-surface phonon scattering. A better understanding for the film boundary scattering of phonons can be found in some recent studies. ${ }^{81-83}$ In one method, Huang et al. ${ }^{59}$ modify the bulk phonon MFPs with $t$ and $L$, given as $1 / \Lambda_{\text {eff }}=1 / \Lambda_{B u l k}+1 / L+2 \cos \theta / t$. Here $\theta$ is the included angle between the phonon traveling direction and the cross-plane direction. This $\Lambda_{\text {eff }}$ is then used in the solid-angle-dependent integration of $k_{L}$.

In this review, a two-step phonon MFP modification is proposed to compute $\Lambda_{e f f}$, which also offers more flexibility in handling independent interface/boundary scattering processes. First, the bulk phonon MFP $\Lambda_{B u l k}$ can be modified as the in-plane phonon MFP $\Lambda_{\text {Fim }}$ for a solid film. Based on the Fuchs-Sondheimer model, $\Lambda_{\text {Finm }}$ is given as ${ }^{78}$

$\frac{\Lambda_{\text {Film }}}{\Lambda_{\text {Bulk }}}=1-\frac{3[1-P(\lambda)] \Lambda_{\text {Bulk }}}{2 t} \int_{0}^{1}\left(x-x^{3}\right) \frac{1-\exp \left(-\frac{t}{\Lambda_{\text {Bulk }} x}\right)}{1-P(\lambda) \exp \left(-\frac{t}{\Lambda_{\text {Bulk }} x}\right)} d x$, where $P(\lambda)$ is the specularity of film-surface phonon reflection. Second, this $\Lambda_{\text {Film }}$ can be modified again to obtain an effective phonon MFP for a nanoporous film, i.e., $\Lambda_{e f f}=\left(1 / \Lambda_{\text {Film }}+1 / L_{e f f}\right)^{-1}$, using the characteristic length $L_{e f f}$ in Fig. 2 for aligned pores. When $L_{e f f}$ is unavailable for the nanoporous pattern, the MBL can always be used but some errors are anticipated. Above two steps for the phonon MFP modification address the phonon size effects for the cross-plane direction and in-plane direction, respectively. Finally, $\Lambda_{e f f}$ is used in Eq. (1) to compute $k_{L}$. In frequency-dependent phonon studies, $k_{L}$ is integrated across the whole phonon spectrum and summed over different phonon branches. Similar two-step phonon MFP modifications from $\Lambda_{B u l k}$ to $\Lambda_{\text {eff }}$ can be found in other studies by Hao et al., such as nanoporous thin films with patterned nanoslots $^{84}$ and nanograined bulk material with nano-inclusions within each grain. ${ }^{85}$ This procedure can be easily extended to general analysis using the exact phonon dispersions and first-principles-computed phonon MFPs.

In demonstration, the analytical model discussed above is used to compute the room-temperature $k_{L}$ for a 220 -nm-thick periodic nanoporous Si film, where MBLs are used as the characteristic length

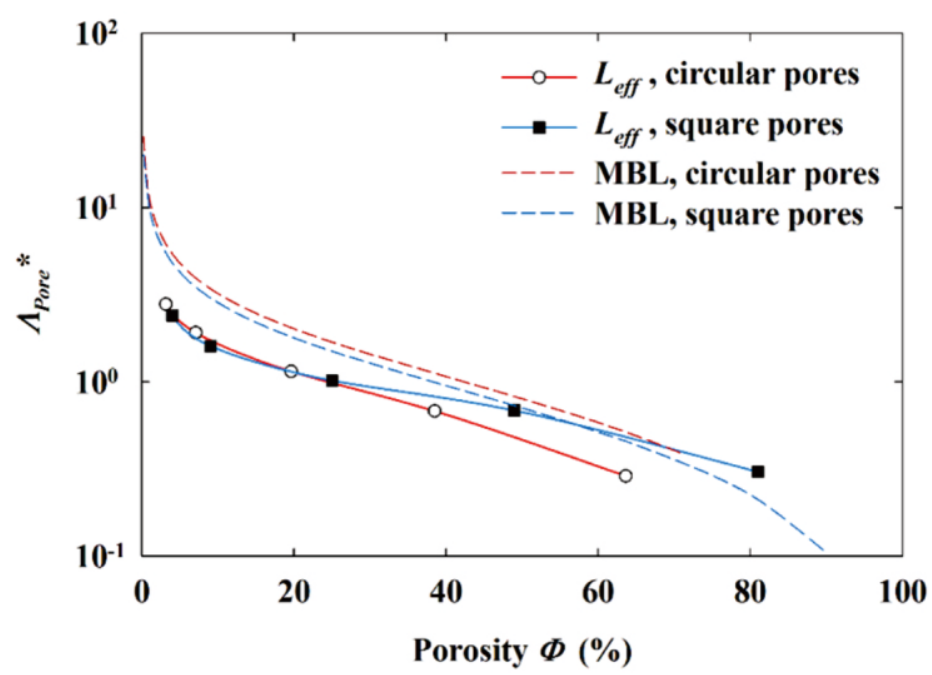

Fig. 2 Porosity- and period-dependent dimensionless $\Lambda_{\text {Pore }}{ }^{*}=L / p$, with $L$ as the MBL or $L_{\text {eff }}$ Summarized from figures in Ref. [45]. Copyright 2016 American Institute of Physics.
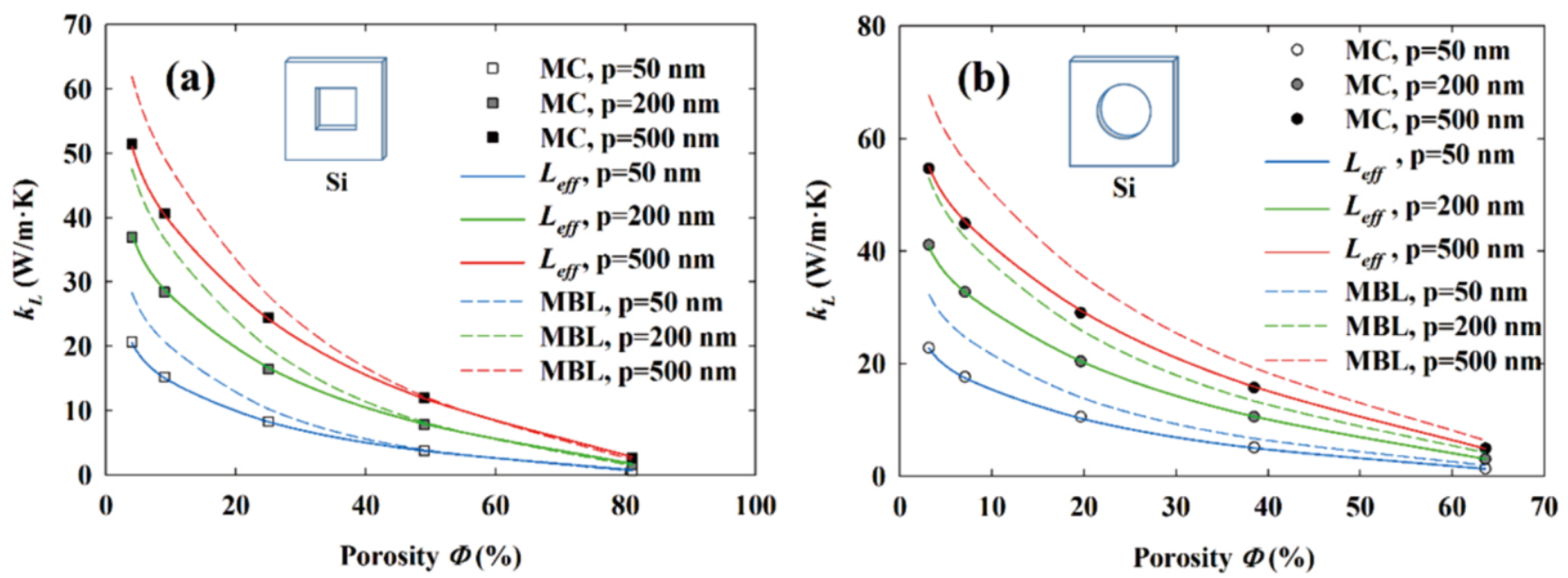

Fig. 3 Predicted room-temperature $k_{L}$ of thin films with aligned pores, using $L_{e f f}$ (solid lines) and the MBL (dashed lines): (a) Square pores in Si, (b) circular pores in Si. The symbols are predictions from frequency-dependent phonon MC simulations to compare with. Reproduced from Ref. [45]. Copyright 2016 American Institute of Physics. 
(Fig. 4a). Completely diffusive phonon reflection is assumed on film surfaces, which is a reasonable assumption above $300 \mathrm{~K}$. In comparison, $k_{L}$ is also predicted by frequency-dependent phonon $\mathrm{MC}$ simulations considering periodic circular through-film pores ${ }^{48}$ (symbols). All calculated $k_{L}$ are normalized by the Hashin-Shtrikman factor ${ }^{61}$ in Eq. (3) to remove the influence of the porosity, yielding $k_{L}$ for the solid or non-porous counterpart of the thin film. Energy-dependent phonon MFPs fitted for bulk $\mathrm{Si}^{86}$ are employed here. It can be observed that the MBL as the characteristic length can slightly overpredict the $k_{L}$. Using the $L_{e f f}$ as the characteristic length instead, the predicted $k_{L}$ becomes consistent with those given by the phonon MC simulations (Fig. 4b).

\subsubsection{Influence of disordered porous patterns}

In most calculations, periodic nanopores are assumed. With completely diffusive phonon reflection by pore edges and thus incoherent phonon transport within the structure, calculations of bulk materials with nanopores showed that $k_{L}$ was insensitive to the spatial configuration and size distribution of the pores. ${ }^{87}$ For general bulk materials with randomly embedded nanoparticles, a similar conclusion was reached, in which the volumetric interfacial area $A / V$ was proposed as one key parameter for the thermal conductivity reduction. ${ }^{88}$ According to the discussion in Section 4.1.2, however, $A / V(1-\Phi)$ should be used as a more accurate parameter to justify the phonon size effects in the host material. Nevertheless, the spatial distribution of pores should not affect the thermal conductivity when phononic effects are negligible. In fact, the $k_{L}$ comparison between ordered and disordered nanoporous Si thin films can be used to evaluate the impact of phononic effects. ${ }^{49,55}$ More discussions are given in Section 4.3.

\subsection{Summary of existing thermal measurements on periodic nanoporous Si thin films}

Table 2 summarizes existing measurements of SOI-based nanoporous thin films, as expanded from a summary given by Marconnet et al..$^{10}$ In nanofabrication, the porous patterns are first defined by photolithography or EBL, 3, 7, 8, 11-13, 48-53, 65, 89 superlattice nanowire pattern transfer technique, ${ }^{14}$ self-assembled block copolymer, ${ }^{33,47}$ or a monolayer film of polystyrene spheres. ${ }^{33}$ Nanopores can then be drilled with reactive ion etching (RIE) or deep reactive ion etching (DRIE). Without any mask, a focused ion beam (FIB) is also employed to directly drill nanopores. $^{2,48}$
Experimentally measured in-plane $k_{L}$ at $300 \mathrm{~K}$ (symbols in Fig. 5) is compared with calculations using $L=\mathrm{MBL}$ and representative film thicknesses ( $t=22 \mathrm{~nm}, 220 \mathrm{~nm}, 2 \mu \mathrm{m}$, and $\infty)$ to modify the bulk phonon MFPs (curves in Fig. 5). First-principles-calculated bulk phonon $\mathrm{MFPs}^{28}$ for $\mathrm{Si}$ are employed for the calculations, which has also been validated experimentally. ${ }^{90}$ For lightly doped $\mathrm{Si}, k \approx k_{L}$ can be assumed. For heavily doped samples ${ }^{14,33,47}$, the electronic $k_{E}$ should be subtracted from $k$ to obtain $k_{L}$. The Wiedemann-Franz law is employed to compute $k_{E}=L_{0} \sigma T$, in which the metallic Lorenz number $L_{0} \approx 2.4 \times 10^{-8} \mathrm{~W} \Omega / \mathrm{K}^{2}$ is approximated for heavily doped samples. ${ }^{78}$ All extracted in-plane $k_{L}$ values are further divided by the Hashin-Shtrikman correction factor in Eq. (3), $F(\Phi)=(1-\Phi) /(1+\Phi)$, to obtain the corresponding solid-film $k_{L}$. To be consistent, other correction factors used in some cited studies are replaced with the Hashin-Shtrikman factor.

In Fig. 5, some extracted $k_{L}$ values are significantly lower than the prediction. Despite some early debates on the possible phononic effects within such samples, 1, 2, 4, 6, 7, 14 now it is often acknowledged that phononic effects should be negligible at $300 \mathrm{~K}$ for all reported samples. ${ }^{29,49,52,65}$ The divergence between measurements and predictions can be attributed to two issues. First, the thermal contact resistance between an employed microdevice and the thin-film sample ${ }^{2,14,33}$ may lead to an overestimated thermal resistance of a sample and thus an underestimated $k_{L}$. Possible damage or distortion may also occur during the transfer process of a nanoporous sample. Such issues were addressed in other studies using an integrated device fabricated from the same $\mathrm{Si}$ film, ${ }^{8,4749} \mu$-TDTR measurements on a suspended sample, ${ }^{3,11,12,51-54}$ and contactless technique of two-laser Raman thermometry., ${ }^{13,55}$ In these studies, the measured $k$ values were mostly comparable to the theoretical predictions at $300 \mathrm{~K}$. Second, more accurate evaluations should also consider the amorphous pore edges introduced by nanofabrication. The significance of pore-edge defects has also been revealed by $\mathrm{MD}$ simulations, where non-propagating modes within amorphous pore edges are further considered. ${ }^{41,91}$ In practice, an effectively expanded pore diameter can be used for thermal analysis. $48,50,92$

In addition to accurate thermal measurements, attention should also be paid to the nanofabrication techniques used to drill the nanopores. The pore-edge defects, which destroys the phase information of coherent phonons, are directly related to the employed nanofabrication techniques. The commonly used pore-drilling techniques include RIE, DRIE and FIB. The surface damage caused by
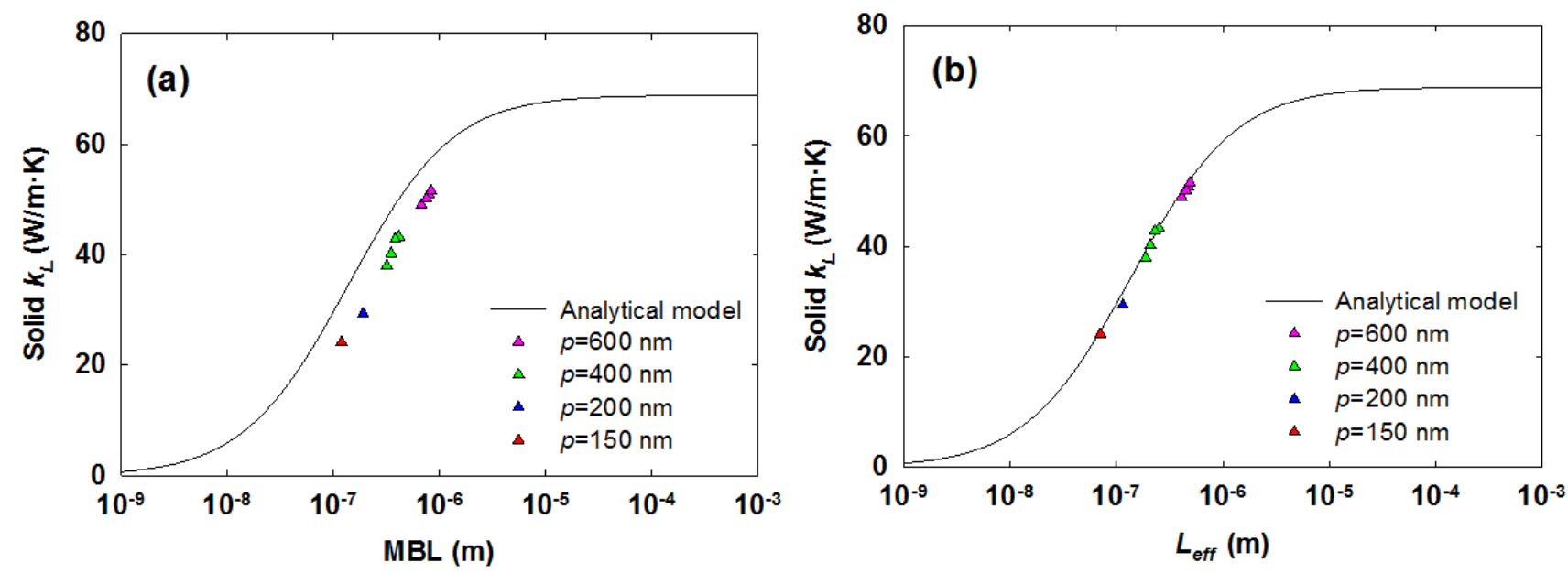

Fig. 4 Comparison between the prediction by the kinetic relationship using modified phonon MFPs (line) and the frequency-dependent phonon MC simulations (symbols) for selected porous patterns across a 220-nm-thick Si film. The employed characteristic length is (a) the MBL and (b) $L_{\text {eff }}$ in Fig. 2. Multiple pore diameters are selected for some pitch $p$. 
Table 2 Thermal measurements of nanoporous Si films. A square (Sq.), staggered (Sta.) or hexagonal (Hex.) lattice for the periodic porous pattern is often used. One paper further compares nanoporous films made of single-crystal and polycrystalline (poly) $\mathrm{Si}^{53}$

\begin{tabular}{|c|c|c|c|c|c|c|c|c|}
\hline Article & Year & $\begin{array}{l}\text { Measurement } \\
\text { configuration }\end{array}$ & Doping & $T(\mathrm{~K})$ & Pattern & $t(\mathrm{~nm})$ & $d(\mathrm{~nm})$ & $p(\mathrm{~nm})$ \\
\hline Hao et al. ${ }^{48}$ & 2018 & $\begin{array}{l}3 \omega \text { technique for a } \\
\text { suspended heater bridge }\end{array}$ & Undoped & $80-300$ & Sq. & 220 & $120-400$ & $150-600$ \\
\hline Anufriev et al.$^{54}$ & 2017 & $\mu$-TDTR & Undoped & $30-296$ & Sq., Sta. & 145 & $80-425$ & $160-500$ \\
\hline $\begin{array}{l}\text { Graczykowski } \\
\text { et al. }\end{array}$ & 2017 & $\begin{array}{l}\text { Two-laser Raman } \\
\text { thermometry }\end{array}$ & Undoped & $300-900$ & Sq. & 250 & $130-140$ & $200-300$ \\
\hline $\begin{array}{l}\text { Yanagisawa } \\
\text { et al. } .^{3}\end{array}$ & 2017 & $\mu$-TDTR & Undoped & 4,295 & $\begin{array}{l}\text { Single row of pores } \\
\text { along } 300 \text {-nm-wide strips }\end{array}$ & 145 & $80-180$ & 300,600 \\
\hline Lee et $_{\text {al. }}{ }^{49}$ & 2017 & $\begin{array}{l}\text { Suspended heater- } \\
\text { thermometer }\end{array}$ & $\begin{array}{l}p \text {-type doped: } \\
10^{15} / \mathrm{cm}^{3}\end{array}$ & $14-325$ & Rectangular holes ${ }^{\text {a) }}$ & $80 \pm 10$ & $60 \pm 5$ & $80-120$ \\
\hline Maire et al..$^{52}$ & 2017 & $\mu$-TDTR & $\begin{array}{l}\text { Boron-doped: } \\
10^{15} / \mathrm{cm}^{3}\end{array}$ & $4-18,300$ & Sq., Disordered ${ }^{\text {b) }}$ & 145 & 133 and 161 & 300 \\
\hline Verdier et al. ${ }^{12}$ & 2017 & $\mu$-TDTR & Undoped & 4,300 & Sq., Sta. & 145 & $90-425$ & $200-500$ \\
\hline Anufriev et al..$^{51}$ & 2016 & $\mu$-TDTR & Undoped & 4,300 & Sq., Hex., Honeycomb & 80 & $65-240$ & $120-280$ \\
\hline Nomura et al. ${ }^{11}$ & 2016 & $\mu$-TDTR & Boron-doped & 300 & Sq., Hex. & 145 & $120-275$ & 300 \\
\hline Wagner et al. ${ }^{13}$ & 2016 & $\begin{array}{l}\text { Two-laser Raman } \\
\text { thermometry }\end{array}$ & Undoped & 300 & Sq., Disordered & 250 & 175 & 300 \\
\hline Nomura et al..$^{53}$ & 2015 & $\mu$-TDTR & $\begin{array}{l}\text { Boron for } \\
\text { single-crystal } \mathrm{Si} ; \\
\text { undoped poly } \mathrm{Si}\end{array}$ & 300 & Sq. & 145,143 & $\begin{array}{l}74-254 \\
\text { (poly Si), } \\
112-270\end{array}$ & 300 \\
\hline Alaie $e t a l .^{2}$ & 2015 & $\begin{array}{l}\text { Suspended island } \\
\text { technique }\end{array}$ & Undoped & 300 & $\begin{array}{l}\text { Sq. w/ smaller } \\
\text { interpenetrating holes }\end{array}$ & 366 & 850 & 1100 \\
\hline Lim et al.$^{47}$ & 2015 & $\begin{array}{l}\text { Suspended heater - } \\
\text { thermometer }\end{array}$ & $\begin{array}{l}\text { Boron-doped: } \\
3.1 \times 10^{18} / \mathrm{cm}^{3} \\
-6.5 \times 10^{19} / \mathrm{cm}^{3}\end{array}$ & $\begin{array}{l}\sim 12- \\
\sim 320\end{array}$ & Hex. & 100 & $26-44$ & $\sim 60$ \\
\hline Kim et al. ${ }^{8}$ & 2012 & Suspended bridge & $\begin{array}{l}p \text {-type boron: } \\
10^{16} / \mathrm{cm}^{3}\end{array}$ & 300 & Sq. & 500 & $204-525$ & $500-900$ \\
\hline $\begin{array}{l}\text { Marconnet } \\
\text { et al. }\end{array}$ & 2012 & Suspended heater bridge & Undoped & 300 & $\begin{array}{l}\text { Single row of pores in } \\
\text { the middle of the } \\
\text { nanobeam }\end{array}$ & 196 & $110-280$ & 385 \\
\hline Hopkins et al. ${ }^{7}$ & 2010 & $\begin{array}{l}\text { TDTR } \\
\text { (cross-plane measurement) }\end{array}$ & $\begin{array}{l}\text { Electrical } \\
\text { resistivity as } \\
37.5-62.5 \Omega \mathrm{cm}\end{array}$ & 300 & Sq. & 500 & $\begin{array}{l}300 \text { and } \\
400\end{array}$ & $500-800$ \\
\hline Tang et al..$^{33}$ & 2010 & $\begin{array}{l}\text { Suspended heater - } \\
\text { thermometer }\end{array}$ & $\begin{array}{l}\text { Intrinsic: } \\
3 \times 10^{14} / \mathrm{cm}^{3} \text {, and } \\
\text { boron - doped: } \\
5 \times 10^{19} / \mathrm{cm}^{3}\end{array}$ & $25-300$ & Hex. & 100 & $32-198$ & $55-350$ \\
\hline Yu et al. ${ }^{14}$ & 2010 & $\begin{array}{l}\text { Suspended heater - } \\
\text { thermometer }\end{array}$ & $\begin{array}{l}\text { Boron-doped: } \\
2 \times 10^{19} / \mathrm{cm}^{3}\end{array}$ & $90-300$ & $\begin{array}{l}\text { Sq. (circular and } \\
\text { square holes) }\end{array}$ & 22 & $\begin{array}{l}11,16 \text { and } \\
270\end{array}$ & 34,385 \\
\hline Song et al..$^{50}$ & 2004 & $\begin{array}{l}\text { Suspended film (steady - } \\
\text { state Joule heating) }\end{array}$ & $\begin{array}{l}n \text {-type: } 5 \times 10^{14} \\
-5 \times 10^{15} / \mathrm{cm}^{3}\end{array}$ & $50-300$ & Sq., Sta. & $4450-7440$ & $\begin{array}{l}1900, \\
2300 \text { and } \\
10900\end{array}$ & $\begin{array}{l}4000 \text { and } \\
20000\end{array}$ \\
\hline
\end{tabular}

\footnotetext{
a) Structure includes periodic and aperiodic rectangular holes.

b) $1 \mathrm{D}$ (single row of holes) and 2D patterns.
} 
these techniques and its impact on the transport properties are acknowledged in the past. In practice, the effective pore diameter can be justified from the $\mathrm{TEM}^{33,48}$ or scanning electron microscopy (SEM) ${ }^{12,52}$ studies. The outer edge of the pore can be treated as the effective pore size. The thermal conductivity reduction due to interface/surface amorphization can also be found in a $\mathrm{Si}$ nanobeam with deposited $\mathrm{Al}$ nanopillars, where the reduced thermal conductivity mainly results from the phonon scattering at the pillar/beam interface with intermixed aluminum and silicon atoms. ${ }^{93}$ George et al. further demonstrated a $30-40 \%$ lower thermal conductivity in silicon membranes covered with aluminum films, leading to significantly enhanced thermoelectric performance. $^{94}$

Fig. 6 shows the measured in-plane $k_{L}$ of four DRIE-drilled samples and two FIB-drilled samples. ${ }^{48}$ Based on the TEM and SEM studies, the pore radius is expanded by $13-40 \mathrm{~nm}$ for DRIE samples with pore diameters of 94 to $300 \mathrm{~nm}$, and $\sim 50 \mathrm{~nm}$ for FIB samples with pore diameters of 200 and $300 \mathrm{~nm}$. Using such effective pore diameters, $k_{L}$ values predicted by frequency-dependent phonon $\mathrm{MC}$ simulations agree well with the measurement results over the whole temperature range.

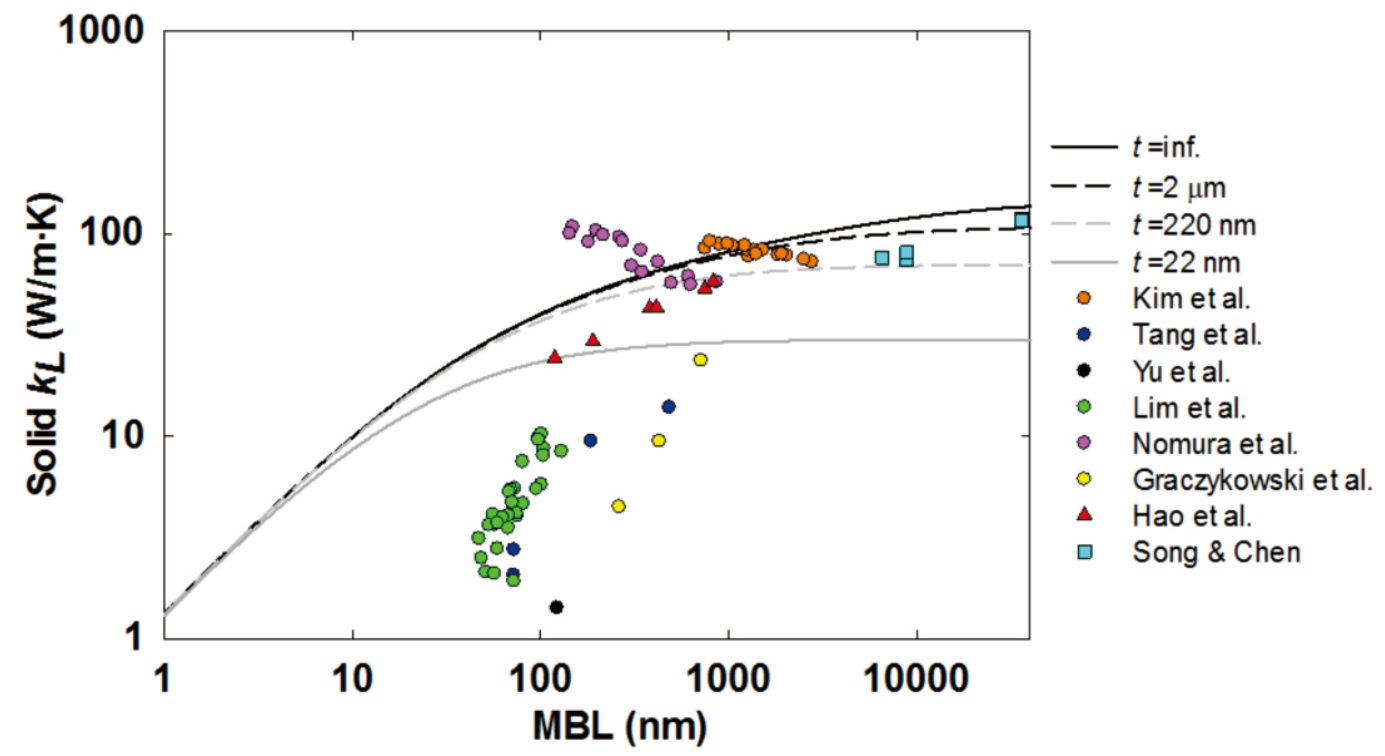

Fig. 5 Comparison between predicted (lines) and measured (symbols) in-plane $k_{L}$ of porous Si films at $300 \mathrm{~K}$. With $F(\Phi)$ corrections, all values are for the corresponding solid film.

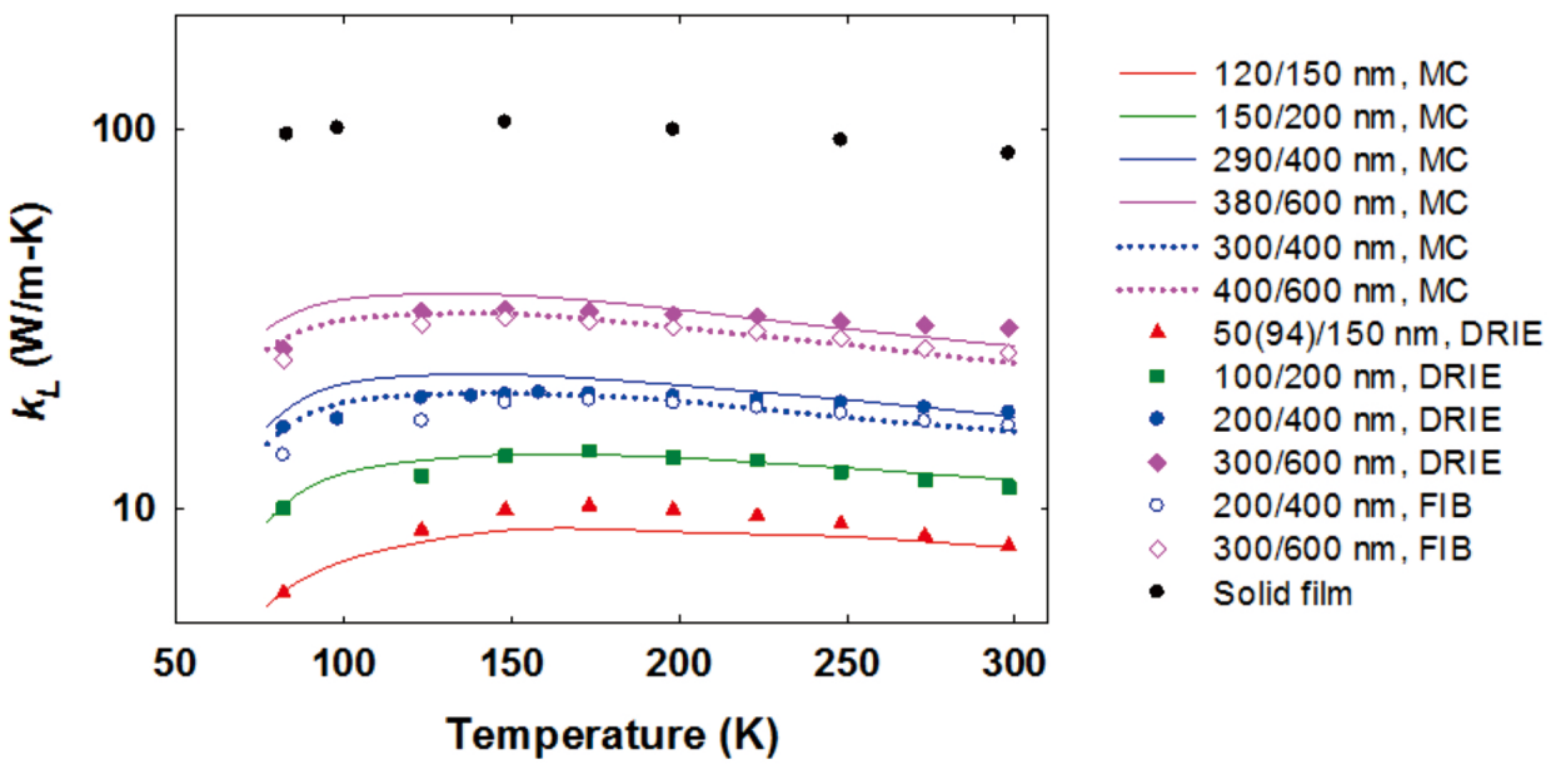

Fig. 6 Temperature-dependent in-plane $k_{L}$ of the solid and nanoporous Si thin film (symbols), in comparison to predictions by frequency-dependent phonon MC simulations (lines with the same color) using an effective pore diameter indicated in the legend. The diameter/pitch combinations are given in the legend. The solid lines are for DRIE samples and the dashed lines are for two FIB samples. No correction factor is used to normalize $k_{L}$. Reproduced from Ref. [48], with Creative Commons Licenses. 


\subsection{Justification of phononic effects within periodic nanoporous thin} films

In practice, phononic effects for phonons are hard to be justified by comparing measurements with predictions assuming completely diffusive pore-edge scattering. This is due to the uncertainties in $k_{L}$ predictions due to the pore-edge defects and employed frequencydependent bulk phonon MFPs. In this section, other experimental methods to justify the phononic effects are discussed in brief.

The first method is based on $k_{L}$ comparison between periodic and aperiodic nanoporous films..$^{13,49,52,55}$ By comparing $k_{L}$ for periodic and aperiodic samples, phononic effects were observed only below $10 \mathrm{~K}$ for nanoporous $\mathrm{Si}$ films with $p=300 \mathrm{~nm},{ }^{52}$ and below $14 \mathrm{~K}$ for $\mathrm{Si}$ nanomeshes with $p>100 \mathrm{~nm}^{49}$ At elevated temperatures, the reduced phonon wavelengths require smaller structures and smoother pore edges so that phononic effects becomes negligible. This finding was consistent with the measurements on Si nanoporous films with $p$ of 200-300 nm, where incoherent phonon transport was found above $300 \mathrm{~K}^{55}$

The second method is to check the possible variation of the phonon dispersion. In one study by Graczykowski et al., the phonon dispersion of nanoporous Si films was measured with Brillouin light scattering. ${ }^{95}$ Wanger et al. used femtosecond pump-probe reflectivity measurements to find the complete Brillouin-zone-center coherent phonon spectrum from the $\mathrm{GHz}$ to the $\mathrm{THz}$ regime. ${ }^{13}$ Along another line, the specific heat of the nanoporous film, as determined by the phonon dispersion, can also be compared with that for bulk $\mathrm{Si}^{48} \mathrm{~A}$ similar heat capacity comparison has also be used to justify the possible phonon dispersion modification for a Si wafer with patterned periodic nanopillars. ${ }^{96}$ In physics, strong phononic effects should lead to strong variation in the phonon specific heat that solely depends on the phonon dispersion. ${ }^{97}$ In contrast, the thermal conductivity is affected by both the phononic effects and pore-edge defects. In early experimental studies, ${ }^{2,3,}$ $7,8,11-14,33,47,49-53,65,89,98$ only the thermal conductivity is measured and no information is available for the specific heat. In this aspect, both the specific heat and thermal conductivity can be measured simultaneously for the same sample using the $3 \omega$ technique for a suspended sample, ${ }^{99}$ providing new insights into the possible coherent phonon transport. Figs. 7a and $7 \mathrm{~b}$ present the solid volumetric specific heat $C$ for all bilayer films patterned with DRIE and a FIB, respectively. All measured $C$ values are divided by $(1-\Phi)$. In general, the solid $C$ values of nanoporous $\mathrm{Si}$ films follow that for a solid film, i.e., a film without nanopores (black dots in Fig. 7a). Some divergence can be attributed to the inaccuracy in $\Phi$ and other defects, considering the wavy pore sidewalls for DRIE samples and tapered sidewalls for FIB samples.

At ultralow temperatures, the bulk phonon MFPs are much longer than $L$ so that $\Lambda_{e f f} \approx L$ can be assumed. In this situation, the temperature dependence of $k_{L} \approx \mathrm{Cv}_{g} L / 3$ is mainly determined by $C$ and thus the phonon dispersion. ${ }^{69}$ Instead of measuring $C$ directly, the comparison of the power law $k_{L} \sim T^{n}$ also indicates the possible change in the phonon dispersion. ${ }^{18}$ In the work by Lee et al. ${ }^{49}$, a clear $k_{L} \sim T^{3}$ trend is observed at low temperature, indicating bulk-like temperature dependence of the specific heat and thus no phononic effects. As one major restriction, this approach is not applicable at $300 \mathrm{~K}$ or above, at which $\Lambda_{e f f}$ and thus $k_{L}$ are further reduced by the temperature-dependent internal phononphonon scattering. In this situation, the temperature dependence of $k_{L}$ does not follow the trend of the specific heat $C$.

\subsection{Direct porous film growth to minimize pore-edge defects}

As discussed above, the pore-edge defects associated with the poredrilling processes can add uncertainties to the phonon transport analysis. When coherent phonon transport is desired, smooth pore edges are critical to the conservation of coherent phonon phases. One way to minimize pore-edge defects is to directly grow nanoporous films with MOCVD, instead of drilling pores after the thin film growth. To block the local growth in porous regions, an array of vertical $\mathrm{SiO}_{2}$ nanopillars can be fabricated on a sapphire substrate as masks. After the film growth at high temperatures, these $\mathrm{SiO}_{2}$ nanopillars can then be etched off with hydrogen fluoride to yield a nanoporous thin film. Roomtemperature phononic effects can be possibly observed if ultrafine nanoporous patterns can be fabricated with this approach.

Fig. 8 shows the measured cross-plane $k$ of nanoporous multilayered films at $300 \mathrm{~K}$, in comparison to theoretical predictions using the MBL and film thicknesses to modify the bulk phonon MFPs within each layer. ${ }^{56}$ Incoherent phonon transport is assumed in the calculations. Each film consists of three layers, i.e., a 50-nm-thick lowtemperature GaN nucleation layer, a 50-nm-thick GaN buffer layer, and a 150 -nm-thick $\mathrm{In}_{0.1} \mathrm{Ga}_{0.9} \mathrm{~N}$ layer. The SEM images of representative samples are shown in the inset, with a fixed $300 \mathrm{~nm}$ pore diameter for all fabricated patterns. The measured $k$ via the TDTR method generally agrees well with the theoretical predictions, with some measurement errors due to the thermal penetration into the substrate. The conclusion here is in contrast with the cross-plane thermal studies of nanoporous $\mathrm{Si}$ films by Hopkins et al., where phononic effects have been suggested to play an important role in the $k$ reduction. ${ }^{7}$ However, the $k$ values of nanoporous films are anticipated to be underestimated by Hopkins et al.
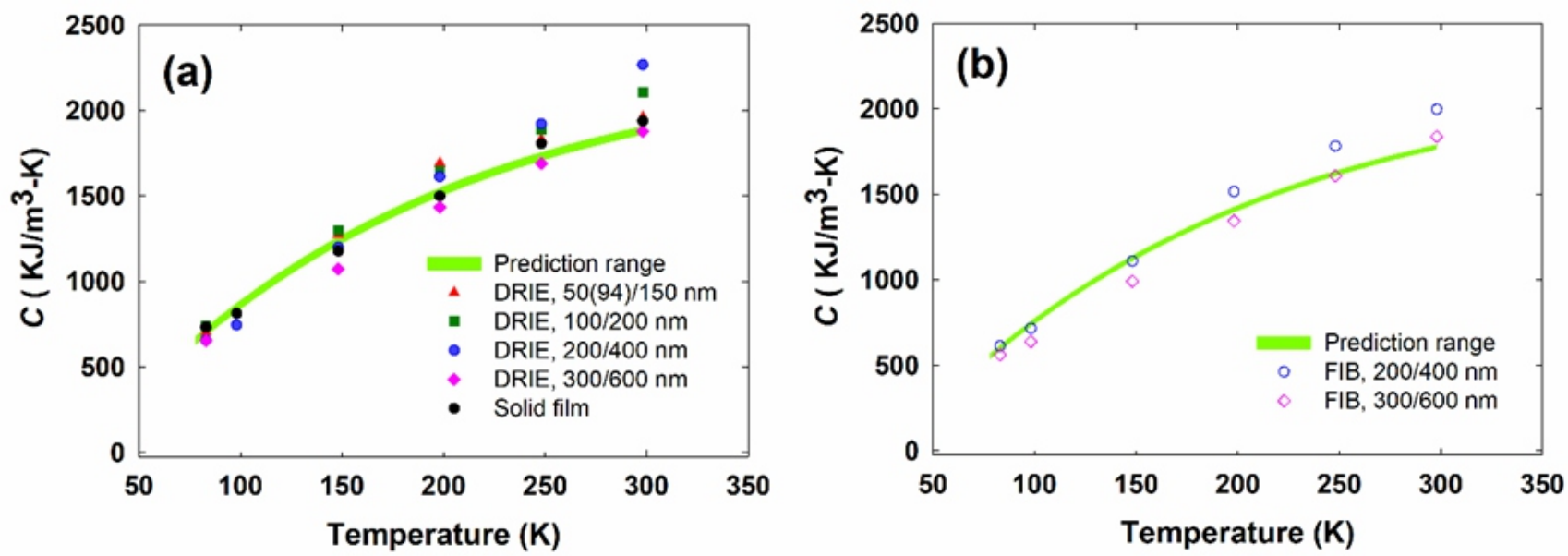

Fig. 7 Temperature-dependent solid $C$ of bilayer films drilled by (a) DRIE and (b) a FIB, in comparison to the prediction using bulk $C$ values for metals and Si. Reproduced from Ref. [48], with Creative Commons Licenses. 
because their calibration of a solid Si film also yields $k$ much lower than theoretical predictions. ${ }^{100,101}$

\section{Thermal studies of antidot lattices (2D phononic crystal)}

With phonon transport is completely constricted within an atomic-thick sheet, periodic porous 2D materials are also studied in recent years though nanofabricated feature sizes are still much longer than the roomtemperature phonon wavelength to induce strong phononic effects at $300 \mathrm{~K}$. Among these materials, GALs (Fig. 1b) have received enormous attention as an effective way to modify the intrinsic transport and optical properties of pristine graphene for applications in electronic/optoelectronic devices, ${ }^{102-104}$ waveguides, ${ }^{105}$ and thermoelectrics. ${ }^{19,} 46,106$ For thermal applications, GALs also belong to "graphene phononic crystals" (GPnCs, shown in Figs. $1 \mathrm{~b}$ and 10), similar to periodic nanoporous $\mathrm{Si}$ films. Compared with thin films with through-film pores, finer porous patterns can be possibly achieved in $2 \mathrm{D}$ materials because the maximum aspect ratio for dry etching does not pose any restriction for atomically thin layers. Numerous calculations have been carried out on GALs with a pitch $p \sim 1 \mathrm{~nm}$ and pore diameter $d<1 \mathrm{~nm}$. For instance, an up to unity room-temperature thermoelectric figure of merit is predicted by Yan et $a l^{46}$ but no experimental validation is available now. In the future, such atomically porous structures can be achieved with advancement in nanofabrication techniques. For graphene, the exact pore-edge configuration, namely armchair and zigzag edges, ${ }^{107}$ is anticipated to affect the phonon scattering as well. The rich physics associated with GALs and other 2D antidot lattices provides new opportunities in exploring phonon transport in a periodic material. Similar thermoelectric studies have also been carried out by Lee et al. on nanoporous Si thin films with the pore size and neck width both in the range of $0.63-2.26 \mathrm{~nm}^{35,}{ }^{36}$ Experimental validation is unavailable for now due to the challenge in fabricating such ultrafine structures.

Experimentally, phononic studies of GALs have major challenges in the nanofabrication of periodic atomic-level pores and accurate thermal measurements. Because $k$ can be largely suppressed by a substrate, ${ }^{108}$ all thermal measurements may be carried out by suspending such fragile materials. The samples can be easily damaged and distorted during the process. In the literature, the only thermal measurement on graphene nanomeshes was carried out by $\mathrm{Oh}$ et al. with a hexagonal pattern. ${ }^{109}$ With a pitch of tens of nanometers, irregular pores were etched across graphene, instead of a highly ordered pattern often assumed in calculations. Using a suspended microdevice, other porous graphene was measured by drilling pores with a FIB across graphene. ${ }^{110}$

${ }^{111}$ In general, above nanoporous patterns, with $\sim 10 \mathrm{~nm}$ or sub-micron pitches, are still too large to compare with superlattices with down to sub-10 nm periodic lengths. Furthermore, highly amorphous pore edges are expected for FIB-drilled patterns, such as an amorphous surface layer of $\sim 10 \mathrm{~nm}$ depth, lattice defects (vacancies, interstitials, and dislocations), Ga ion implantation, and large atom displacement within the collision cascade that extends tens of nanometers from the cut surface. ${ }^{112}$ In this aspect, scanning helium ion lithography can be better for the nanofabrication of $2 \mathrm{D}$ materials. ${ }^{113}$ For a suspended graphene sample, sub-1 $\mathrm{nm}$ pores can be directly patterned with focused electron beams under a TEM. ${ }^{114}$ However, the high-energy electrons also cause defects or structural changes, shown as a concentric ring-like structure around pores. For phononic studies, these pore-edge defects must be considered.

\subsection{MBL of 2D nanoporous materials}

Different from thin films, the phonon boundary scattering by the top and bottom surfaces is eliminated in suspended 2D materials to simplify the analysis. For GALs, ballistic electron transport is suggested because the structure size is often much shorter than majority electron MFPs in pristine graphene. ${ }^{115,116}$ The same conclusion may also be anticipated for phonons in graphene so that $k_{L}$ can be computed assuming $\Lambda_{e f f} \approx M B L$ for all phonons. This argument is known as the small-nanostructure-size limit, where $\Lambda_{e f f}$ approaches the characteristic length of nanostructures. ${ }^{117}$ ${ }^{118}$ The existing derivation of the MBL for a $3 \mathrm{D}$ enclosure $^{119}$ can be extended to a $2 \mathrm{D}$ enclosure and used for phonon modeling. Due to the decreased degrees of freedom for phonon movement, phonons travel a shorter distance between their successive collisions with pore edges, leading to a shorter 2D MBL than quasi-2D MBLs given in Eq. (4). In this review, the existing MBL derivation for a $3 \mathrm{D}$ enclosure $^{119}$ is

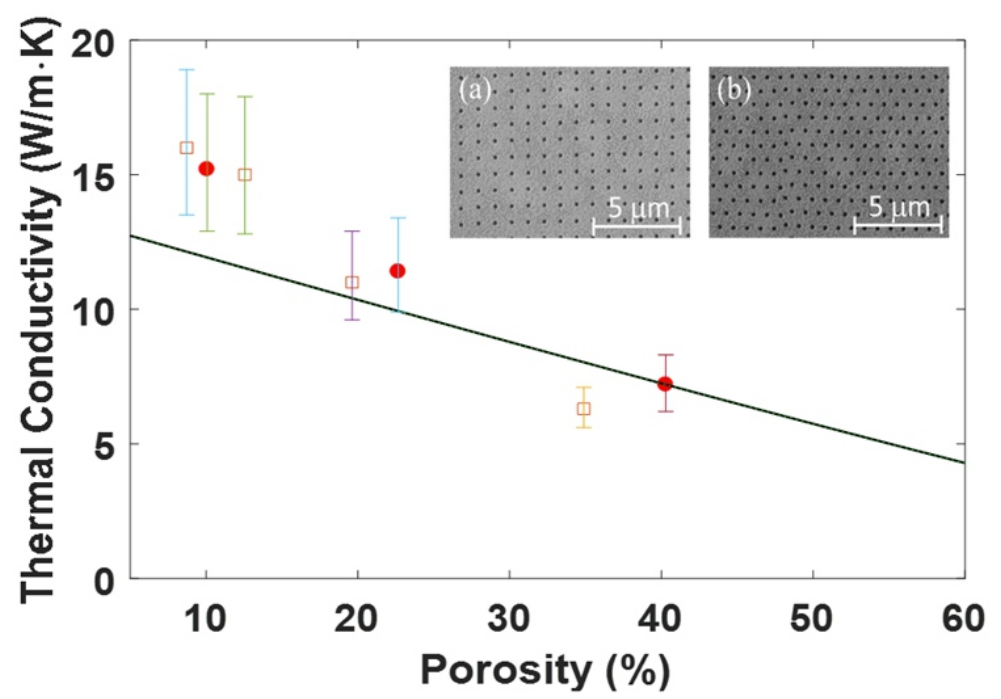

Fig. 8 Comparison between the measured and predicted $k$ values for tri-layered nanoporous GaN-based films. Here filled circles are for hexagonal patterns, whereas empty squares are for patterns on a square lattice. The insets show the SEM images for representative samples. Reproduced from Ref. [56]. Copyright 2018 Springer Nature. 
extended to a pure 2D enclosure.

Within a plane, consider an isothermal and nonscattering medium that is surrounded by a $2 \mathrm{D}$ blackbody enclosure with perimeter $P$ (Fig. 9a). In such an enclosure, the spectral heat flux onto any point on the wall is given by the integration of incident directional intensity $I_{b m}$, where the subscript $b$ indicates blackbody and $\eta$ is the wavenumber. The integration is over the hemispherical solid angle $\Omega=2 \pi$ for a 3D enclosure, and is over a half-circle solid angle $\Omega=\pi$ for a $2 \mathrm{D}$ enclosure. The wavenumber-dependent heat flux $q_{\eta}$ follows the same expression as a $3 \mathrm{D}$ enclosure ${ }^{119}$ except for the change in the solid angle integration:

$$
\begin{aligned}
q_{\eta} & =I_{b \eta} \int_{\text {Half circle }}\left(1-e^{-\kappa_{\eta} L_{d}(\hat{s})}\right) \cos \theta d \Omega \\
& =2 I_{b \eta} \int_{\theta=0}^{\frac{\pi}{2}}\left(1-e^{-\kappa_{\eta} L}\right) \cos \theta d \theta \\
& =2 I_{b \eta}\left(1-e^{-\kappa_{\eta} L}\right),
\end{aligned}
$$

in which an effective length $L$ as the MBL replaces the directiondependent distance $L_{d}(\hat{S})$ from this point to the wall along the $\hat{S}$ direction (Fig. 9b). When $\kappa_{\eta} L \rightarrow 0$ for a optically thin medium, Eq. (6) becomes

$$
q_{\eta} \approx 2 I_{b \eta} \kappa_{\eta} L .
$$

On the other hand, total radiation received by the wall along its perimeter, $q_{\eta} P$, is from the blackbody emission inside the whole medium-filled area $S$ within the 2D enclosure. For an optically thin medium, no self-absorption occurs and $q_{\eta} P$ is given as

$$
q_{\eta} P=\left(2 \pi I_{b \eta}\right) S \kappa_{\eta} .
$$

In the derivation of 3D MBLs, $q_{\eta} A=\left(4 \pi I_{b \eta}\right) V \kappa_{\eta}$ is instead suggested for a 3D enclosure with inner wall surface area $A$ and filled volume $V{ }^{119}$ Comparing Eqs. (7) and (8) gives the effective MBL as

$$
L=\frac{\pi S}{P}
$$

For nanoporous 2D materials, $S$ corresponds to the solid area $S_{\text {Solid }}$ of a period and $P$ is the pore perimeter. By fitting the MCRT results for a $2 \mathrm{D}$ material with aligned pores, $\mathrm{L} \approx 3.4 S_{\text {Solid }} / P$ is obtained and is close to the $L$ expression in Eq. (9).

\subsection{Existing thermal studies of antidot lattices}

Currently, measurements on GALs are focused on their electrical properties. $^{70,102,115,116,120}$ Instead of GALs with aligned pores, thermal measurements are only available on graphene nanomeshes with irregular pores. ${ }^{109}$ This study employed the optothermal Raman technique, where a Raman laser was used to heat up a sample suspended across a hole and the associated temperature rise was read from the Raman peak shift. ${ }^{121,122}$ The effective $k$ of a GAL can thus be obtained from 2D heat conduction analysis. Such measurements often have large uncertainties due to 1) the difficulty in determining the actual laser power absorbed by a sample and 2) complexity introduced by the strong thermal nonequilibrium between electrons, optical phonons and acoustic phonons inside graphene. ${ }^{123}$ More comparison measurements with accurate measurement techniques should be carried out to better understand the coherent and incoherent phonon transport within periodic nanoporous structures.

Different from real samples with inevitable defects, atomistic simulations assuming periodic atomic structures often suggest much stronger phononic effects. In simulations and modeling, it is found that the thermal conductivity of a GAL can be significantly lower than that of graphene and can be efficiently tuned by changing the porosity and period length. ${ }^{115}$ Further phonon mode analysis reveal that the thermal conductivity reduction is due to the increasing of phonon localization and three-phonon scattering in a GAL. ${ }^{124}$

Instead of drilling pores, direct bottom-up synthesis of nanoporous graphene $^{125}$ can be more ideal for phononic studies. With an ordered inclusion of uniform holes and nitrogen atoms, $\mathrm{C}_{2} \mathrm{~N}-\mathrm{h} 2 \mathrm{D}$ crystals were also synthesized. ${ }^{126}$ Other periodic $2 \mathrm{D}$ structures included polyaniline $\left(\mathrm{C}_{3} \mathrm{~N}\right)$ with six nitrogen atoms periodically surrounding a phenyl ring. ${ }^{127}$ By introducing a periodic array of zigzag holes into the monolayer $\mathrm{C}_{3} \mathrm{~N}$ structure, the formed phononic crystal was studied by non-equilibrium MD simulations for the impact of disordered pore distributions. ${ }^{42}$

\subsection{Phonon local resonance effects and thermal rectification applications}

Instead of drilling pores, introducing periodic pillars on two sides of graphene nano-ribbon to form graphene nano-ribbon with nano cross junctions (NCJs) (Fig. 10) will induce phonon local resonant hybridization, which also greatly reduces the thermal conductivity. This new type of phononic crystals is intrinsically different from those using the interference between coherent phonon modes within periodic nanoporous materials. Also called "nanophononic metamaterials" and (a)

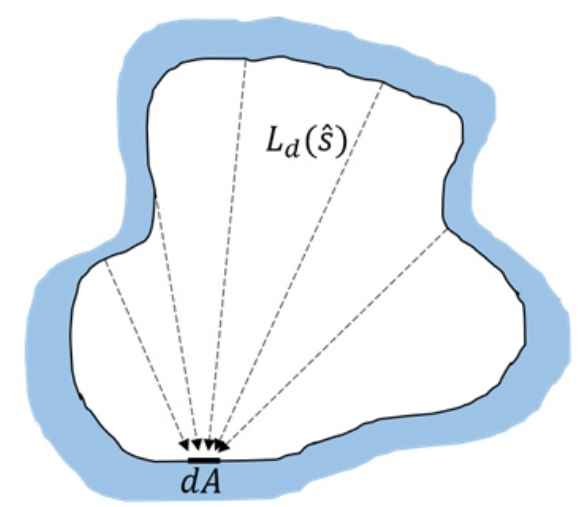

(b)

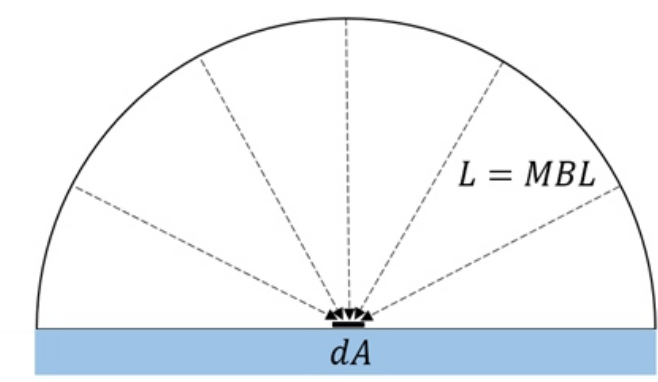

Fig. 9 (a) An arbitrary 2D enclosure filled with an isothermal and nonscattering medium. (b) Equivalent half circle radiating to the center of its base as the receiving point in (a). 
sometimes "phonon resonant structure," such pillar-modified structures were proposed by Nika ${ }^{128}$ and Davis et al. ${ }^{129}$ Extensive studies can be found elsewhere. ${ }^{96,130-135}$ As an interesting result in simulations, the thermal conductivity of graphene nano-ribbon with NCJs increases after replacing $\mathrm{C}^{12}$ in pillars with isotopic atoms (lighter or heavier than $\mathrm{C}^{12}$ ). It is caused by the mismatch between resonant modes and propagating modes, which breaks and decreases the original hybridization and facilitates phonon transport. ${ }^{136}$ Again it should be noted that these MD simulations still require experimental validation, as defects in real samples may largely decrease the predicted wave effects. In measurements, a reduced thermal conductivity of a Si nanobeam with deposited Al nanopillars was observed. ${ }^{93}$ However, this thermal conductivity reduction was attributed to the intermixing of $\mathrm{Al}$ and $\mathrm{Si}$ atoms at the pillar/beam interface, instead of the wave effects. In principle, metallic pillars are not preferred to produce strong local phonon resonance because the dominant heat carriers in metals are electrons instead of phonons. Other than pillar-modified structures, thermal conductivity suppression due to phonon resonance can also be found in $\mathrm{Ge} / \mathrm{Si}$ core-shell nanowires ${ }^{137-139}$ and in host-guest systems with hybridized modes originating from guest atoms. ${ }^{140,141}$

In addition to the decreased thermal conductivity, the temperature dependence of thermal conductivity for graphene can also be modulated. Compared to pristine graphene, the thermal conductivity of GALs (e.g., Fig. $1 \mathrm{~b}$ for hexagonal porous patterns) has a weaker dependence on temperature, $k \sim T^{-\alpha}$. The power exponent $(\alpha)$ can be efficiently tuned by changing the characteristic size of such phononic crystals. ${ }^{142}$

A potential application of GAL is for thermal rectification. It is found that the asymmetric graphene/GAL system is a promising thermal rectifier, ${ }^{143}$ because of the different temperature dependence of thermal conductivities in graphene and GAL. It is also observed in experiments. ${ }^{110} \mathrm{~A}$ thermal rectification factor of $26 \%$ is achieved in a defect-engineered monolayer graphene with nanopores on one side. ${ }^{110}$ This remarkable thermal rectification results from the difference in temperature dependence of $k$ for graphene and GAL regions, which can be regarded as nonlinearity introduced into the system. ${ }^{144}$ Anticipated to be weak around $300 \mathrm{~K}$ for FIB-drilled graphene, ${ }^{145}$ phononic effects are not required to achieve this difference. To enhance the thermal rectification ratio, a series circuit of thermal rectifiers is proposed. ${ }^{143} \mathrm{Hu}$ et al. studied thermal rectification in both two-section and three-section asymmetric graphene/GAL structures. Similar to the series effect in electronic circuits, the series effect is demonstrated by the consistence between the results of theoretical prediction and that of MD simulations. It is found that both the series effect and size effect are effective strategies to enhance the thermal rectification ratio.

\section{Porous nano-cages (3D phononic crystal)}

$3 \mathrm{D}$ periodic materials have attracted great attention due to their potential application in thermoelectrics. Compared to $1 \mathrm{D}$ and 2D structures, a 3D

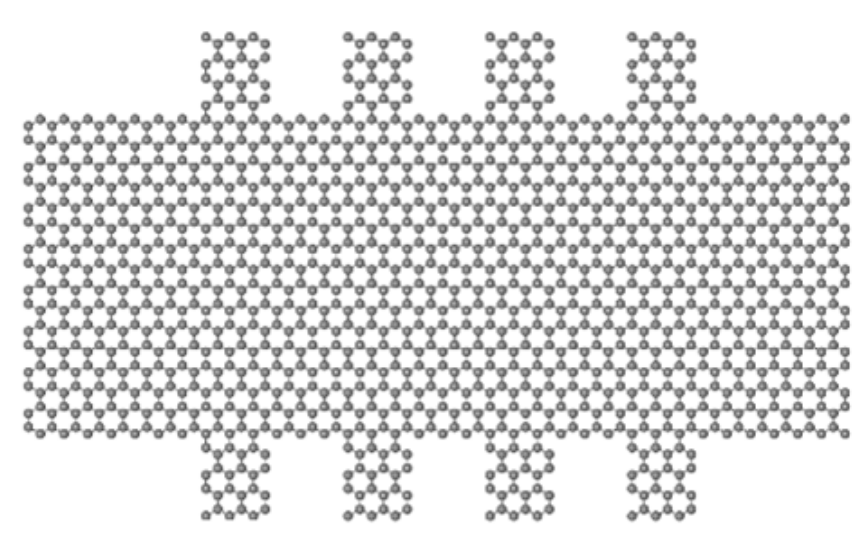

Fig. 10 Graphene nano-ribbon with NCJs.
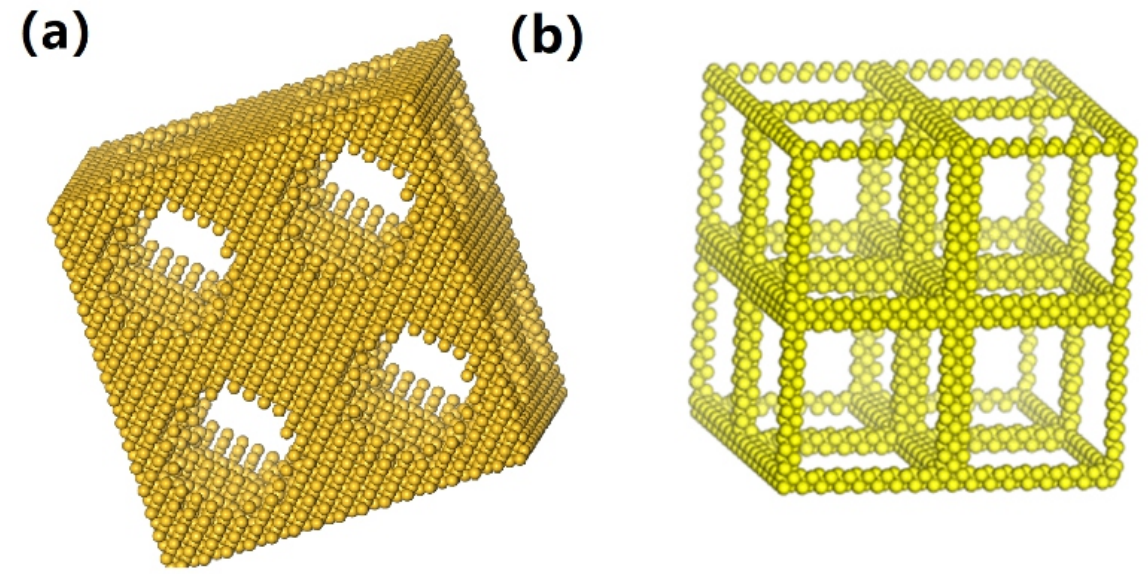

(c)

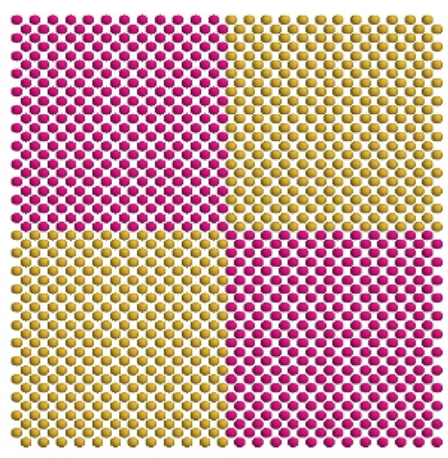

Fig. 11 The structure of a (a) periodic spherical porous 3D phononic crystal, (b) SiNW-cage and (c) isotopic 3D Si phononic crystal. 
phononic crystal is bulk and are more suitable for common applications. Periodic spherical porous 3D Si phononic crystal (Fig. 11a), SiNW-cage structure (Fig. 11b) and isotopic 3D Si phononic crystal (Fig. 11c) are studied. The Si phononic crystal is constructed by periodic arrangement of nanoscale supercells constructed from a cubic cell with a spherical pore. The SiNW-cage consists of SiNWs connected by NCJs. The isotopic 3D $\mathrm{Si}$ phononic crystal is assembled periodically in three directions by ${ }^{28} \mathrm{Si}$ and ${ }^{\mathrm{M}} \mathrm{Si}$ atoms. For these structures, MD simulations are again used and wave effects are overestimated due to the assumed perfect periodic structures. Experimentally, it is still challenging to fabricate these 3D nanoporous structures with smooth surfaces.

Phonon wave effects can effectively reduce the thermal conductivity of a $\mathrm{Si}$ phononic crystal, SiNW-cage structure and isotopic 3D $\mathrm{Si}$ phononic crystal. It is found that the thermal conductivity of a $\mathrm{Si}$ phononic crystal is decreased to $0.022 \mathrm{~W} / \mathrm{m} \cdot \mathrm{K}$, which is only $0.01 \%$ of the thermal conductivity for bulk Si. This low thermal conductivity decreases as the porosity increases. The reduction in the thermal conductivity is due to more phonons localized in a $\mathrm{Si}$ phononic crystal at boundaries. ${ }^{37}$ In contrast to the huge thermal conductivity reduction, the electronic transport coefficients of a Si phononic crystal at $300 \mathrm{~K}$ are reduced slightly, and the Seebeck coefficient is similar to that of bulk Si. This leads to a higher ZT $=0.66$ of a Si phononic crystal, which is about 66 times of that of bulk $\mathrm{Si}^{38}$ For SiNW-cage structures, the thermal conductivity can be as low as $0.173 \mathrm{~W} / \mathrm{m} \cdot \mathrm{K}$, which is even one order of magnitude lower than that of $\mathrm{SiNW}^{39}$ The large reduction in thermal conductivity is due to significant phonon local resonant hybridization at the junction part which reduces group velocity in a wide range of phonon modes. ${ }^{39}$ The mechanism here are not excepted to scatter electrons, which will increase the ZT. For an isotopic 3D Si phononic crystal, the thermal conductivity is significantly reduced at $1000 \mathrm{~K}$. Increasing the mass ratio will further decrease the thermal conductivity. The decrease of thermal conductivity in a isotopic 3D Si phononic crystal is attributed to both the decrease of group velocities and the localization. ${ }^{40}$

\section{Quantifying phonon particle and wave transport in 3D phononic crystals}

In periodic structures with smooth boundaries or interfaces, the mix of wave and particle phonon transport is expected though phonon wave transport can dominate. During the past decades, many researches have focused on quantitative understanding of particle and wave transport of electrons and photons. ${ }^{146,}{ }^{147}$ In order to optimally manipulate phonon transport, the direct individual contributions of phonon particle and wave effects to the modulation of thermal conductivities need to be evaluated.

Phonon particle and wave effects can be quantified by combining MC and AGF methods. ${ }^{148}$ To probe phonon transport from the particle standpoint, the MC method can be used for a NCJ system. The transmittance can be used to decide whether phonons can transport across the junction part (Fig. 12a). The phonon wave information is included in this transmittance. To incorporate the transmittance into the $\mathrm{MC}$ simulation, a random number is drawn from a uniform distribution for every phonon. By comparing this random number with the transmittance, we decide whether the phonon can transport across the junction part or not. Here, the AGF method is used to obtain the transmittance. The combination of the AGF and MC methods is termed as AGFMC. As shown in Fig. 12b, with the introduction of cross junction, $k_{\text {NCIMC }}$ (blue dot) is smaller than $k_{S i N W}$ (black dot). This is because the junction increases the phonon scattering. In addition, $k_{\text {NCJ AGFMC }}$ (red dot) is even smaller than $k_{\text {NCJMC }}$ (blue dot). This is due to the enhanced blockage originating from phonon resonant hybridization as wave effects, which has been further incorporated into the AGFMC. The fraction of thermal conductivity reduction by wave effects to the total thermal conductivity reduction is shown in Fig. 12c. When the cross section area (CSA) increases from $2.23 \mathrm{~nm}^{2}$ to $17.72 \mathrm{~nm}^{2}$, the wave ratio $\left(\eta_{\text {wаve }}\right)$, which measures the fraction of thermal conductivity reduction by the wave effect to the total thermal conductivity reduction, decreases monotonously. This shows that the wave effect weakens as the system size increases, which is in accordance to previous studies. What is more striking, as shown in Fig. 12c, the wave effect is only $68 \%$ (corresponding to $32 \%$ particle effects), even for the CSA as small as $2.23 \mathrm{~nm}^{2}$ for 4-leg NCJs (shown in the inset of Fig. 12c). This accentuates the importance of mutually controlling phonon particle and wave characteristics in NCJ.

In periodic nanoporous structures, wave effects are anticipated for some long-wavelength phonons and particle view should be valid for phonons with very short wavelengths. When wave effects become notable with a reduced periodic length and/or at cryogenic temperatures, the two phenomena can coexist. Such particle and wave phonon
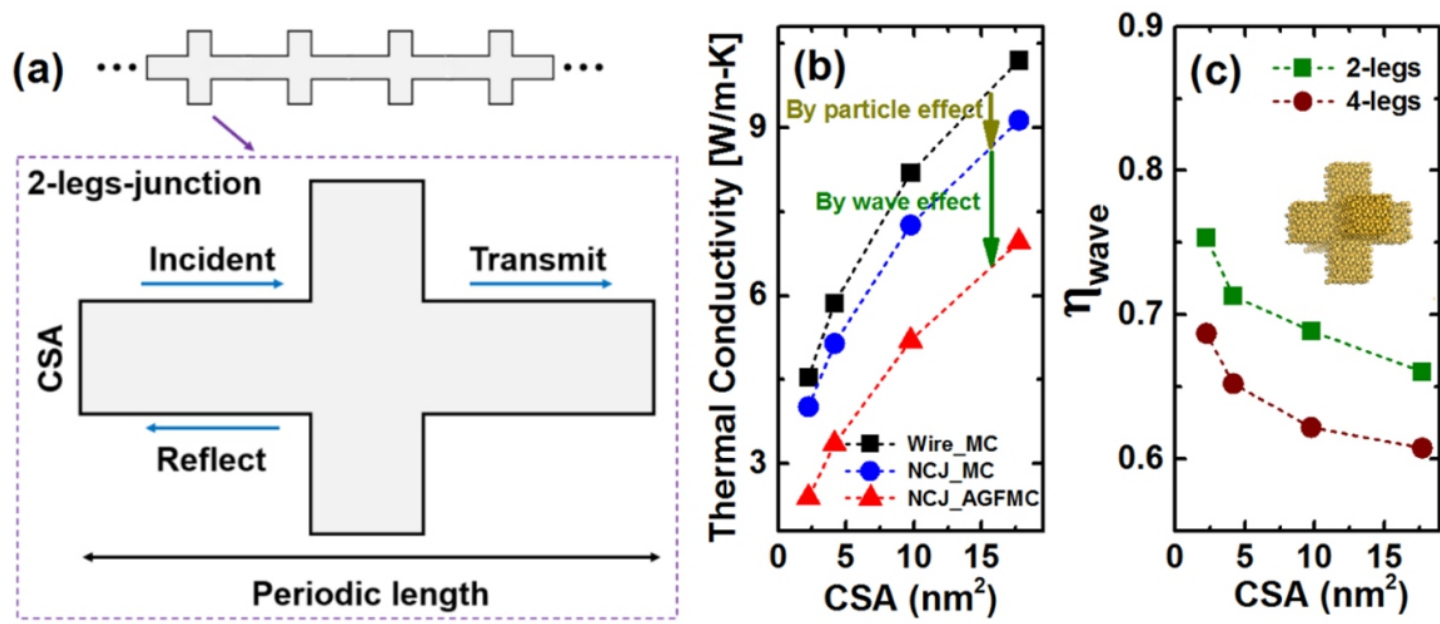

Fig. 12 (a) Schematic picture of phonon transport across a NCJ system. (b) The thermal conductivity of a SiNW (black dot) and NCJ (red and blue dot) as a function of the CSA at $300 \mathrm{~K}$. The data of the red line are obtained by MC, which only takes the phonon particle effect into account. The data of the blue line are obtained by the AGFMC, which considers both phonon particle and wave effects. (c) The ratio of the thermal conductivity reduction by the phonon wave effect to the total thermal conductivity reduction versus the CSA. 
conduction can be quantified by a two-phonon model, ${ }^{149,150}$ which can be used for quantifying wave conduction in periodic phononic structures. But the two phonon model considers all phonons as two gray media. The fact that different phonon modes can have different wavelengths is significantly neglected, and the non-equilibrium between phonons are not considered. ${ }^{151}$ In earlier studies on superlattices, a modified lattice dynamics model has been proposed, in which incoherent interfacial phonon scattering is incorporated with a complex wave vector involving the phonon MFP. $^{30,152}$ In one recent work, an improved phonon MC technique has been proposed, where a particle model can further include wave properties to model interferences between wave packets. ${ }^{153}$ This technique is suitable for periodic structures with length scales larger than phonon coherence length (e.g., $\sim 1 \mathrm{~nm}$ at $300 \mathrm{~K}$ for $\mathrm{Si}^{7^{8}}$ ), where coherent phonon transport may still take place in structures such as GaAs/AlAs superlattices. ${ }^{154}$

\section{Summary and perspective}

Similar to photonic crystals, phononic crystals based on periodic structures provide new opportunities in manipulating lattice vibrational waves. To have a strong influence on heat conduction by wave effects, two conditions must be satisfied: 1) the pitch should be comparable to the majority phonon wavelengths but much shorter than phonon MFPs; 2) the interface/surface roughness should be smaller than the phonon wavelength to minimize the diffusive phonon scattering that destroys the phase information of phonons. The influence of more complicated mechanisms, such as the Akhiezer mechanism, ${ }^{15}$ may also be considered in detailed studies. This mechanism is due to the coupling of the strain of sound waves and thermal phonons. In practice, the Akhiezer mechanism can largely suppress the contribution of low-frequency phonons that are more likely to be affected by the phononic effects. ${ }^{15}$

In $\mathrm{Si}$, majority phonon wavelengths at $300 \mathrm{~K}$ are of $1-10 \mathrm{~nm}^{28,29,155}$ Therefore, the reported room-temperature phononic effects ${ }^{2,7}$ in nanoporous structures can be mostly attributed to measurement errors and other structural defects such as amorphous pore edges. Phononic effects only become critical at around $10 \mathrm{~K}$ or below for nanoporous $\mathrm{Si}$ films, when heat conduction is dominated by phonons with wavelengths of tens of nanometers. However, such ultra-low-temperature phononic crystals have very limited applications. Compared with nanofabricated porous materials with $p$ as tens of nanometers or longer, superlattice thin films can achieve sub-10 $\mathrm{nm}$ periodicity and atomically smooth interfaces, offering more opportunities in phononic studies. For 2D materials, the aspect ratio restriction for pore-drilling techniques is not applicable and sub-10 nm pitches can be potentially fabricated with EBL patterning. For suspended samples, both a FIB and electron beams can be directly used for the drilling. However, varied pore-edge defects can be induced and may diminish the coherent phonon transport.

Without pore drilling, direct growth of nanoporous films ${ }^{56}$ or $2 \mathrm{D}$ materials with patterned pillar-like masks can minimize pore-edge defects to potentially yield a comparable case as superlattice thin films. For thermal management and thermoelectric applications, atomic-thick 2D materials are restricted by their low in-plane thermal and electrical conductances. Challenges also exist in the accurate thermal measurements of 2D structures. ${ }^{156}$ In practice, ultrafine nanoporous thin films can be more useful. In this aspect, direct MOCVD growth may achieve a much higher aspect ratio for the through-film pores, compared with $t / d<3$ for dry-etched films. The thickness of the grown film can be possibly larger than the height of the mask. Using only 40-nm-thick nanoporous $\mathrm{SiO}_{2}$ as a mask, vertical micrometer-length $\mathrm{GaN}$ nanowires with a $50 \mathrm{~nm}$ diameter can be grown. ${ }^{157}$ Considering the $\sim 5 \mathrm{~nm}$ spatial resolution for the state-of-the-art EBL, sub-10 nm masks can thus be fabricated to directly grow ultrafine nanoporous thin films with a thickness of tens of nanometers. The minimum $k$ predicted for superlattices $^{26,152}$ is anticipated for ultrafine porous films or 2D materials with smooth pore edges, resulting from the interplay of periodicity for coherent phonon transport and pore-edge defects for incoherent phonon transport.

Existing studies about phononic crystals are proposed for thermoelectric applications, in which bulk-like electrical properties can be maintained but the thermal conductivity $k$ can be dramatically reduced. $^{14,33,46}$ Other than thermoelectrics, heat guide and phonon focusing are also studied by patterning designed nanoporous regions across a Si film. ${ }^{54}$ When impurity atoms are further introduced to scatter the shortwavelength phonons, it is possible to narrow down the phonon conduction to the middle-wavelength regime. This so-called thermocrystal, ${ }^{9,} 17$ are proposed for heat waveguides, thermal lattices, heat imaging, thermooptics, thermal diodes, and thermal cloaking.

Besides aligned or staggered circular pores, other porous patterns are also studied under the circumstance of incoherent phonon transport. ${ }^{158,159}$ When the porous patterns are designed to tune the phonon transmission by pore-edge phonon scattering, asymmetric phonon transmission along the forward and backward directions may lead to thermal rectification effects. ${ }^{71,160}$ The high performance of such a thermal rectifier requires strong ballistic transport within the nanoporous pattern and strong specular reflection by pore edges. The two requirements can be mostly satisfied at low temperatures, where dominant phonon MFPs become much longer than the structure size and the phonon wavelengths become long compared with the pore-edge roughness to ensure specular phonon reflection. Following this, Schmotz et al. reported thermal rectification effect at $150 \mathrm{~K}$ for designed porous patterns though this effect was not found at $300 \mathrm{~K}{ }^{161}$ However, no thermal rectification effect beyond the instrumental uncertainty was found by Gluchko et al. at 4.2-300 K in Si films with Pacman pores ${ }^{162}$ and by Kage et al. at 5-295 K in Si films with dog-leg-shaped pores. ${ }^{163}$ In physics, Maznev et al. ${ }^{164}$ argued that nonlinearity must be introduced into a system and asymmetric structure alone was not sufficient to induce thermal rectification. Different from a ballistic thermal rectifier, thermal rectification was reported by Wang et al. for suspended graphene with one end modified by nanostructured created with electron-beam-induced deposition or FIB drilling. ${ }^{110}$ Thermal rectification was simply caused by the contrast in the temperature dependence of $k_{L}$ for the unpatterned and nanostructure-patterned regions. The total thermal conductance of the sample was thus different when the hot and cold ends were switched. ${ }^{144}$

In general, a better manipulation of the phonon wave effects also requires well-designed nanostructures. Luckyanova et al. found that coherent phonon transport was dominant in GaAs/AlAs superlattices at $30-150 \mathrm{~K}$ and was still important even at $300 \mathrm{~K} .^{154}$ In principle, coherent phonons have low frequencies and long wavelengths. ${ }^{149,154}$ These phonons are less affected by interfacial defects but can be strongly scattered by ErAs dots covering the interface area. ${ }^{165}$ When these dots as scattering centers are randomly distributed at the interfaces, the destructive phonon interference can lead to phonon localization to suppress the transport of low-frequency phonons. In practice, the density and/or the randomness degree of these ErAs dots can be better designed, which can benefit from materials informatics. ${ }^{166,167}$

In summary, coherent and incoherent phonon transport in periodic porous or further pillar-modified structures can play an important role in the manipulation of thermal transport within materials and devices. Despite numerous atomistic simulations, ultrafine periodic structures down to the atomic level are still hard to be fabricated and the amorphous pore edges often destroy coherent phonon phases. In experiments, coherent phonon transport and thus phononic effects can 
only be notable around or below $10 \mathrm{~K}$, which largely restricts the applications of these materials. In this aspect, future work should be carried out on fabricating and measuring ultrafine nanoporous structures with minimized pore-edge defects. With such structures, existing designs of optical and acoustic devices can be adopted by phononic devices to manipulate phonons. Without phononic defects, classical phonon size effects are considered for such porous structures but the pattern shape and distribution can still be varied to benefit many applications. $^{71,158}$ In general, certain "thermally dead volume" can be introduced in a patterned thin film, where phonons can be trapped and contribute less to heat conduction. Examples can be found in nanoladders with a row of rectangular holes ${ }^{159}$ and SiNWs with periodic wings. ${ }^{168}$

\section{Acknowledgements}

Q. H. acknowledges the support from the U.S. Air Force Office of Scientific Research (award number FA9550-16-1-0025) for studies on nanoporous materials and National Science Foundation (grant number CBET-1651840) for phonon simulations. N.Y. was sponsored by National Natural Science Foundation of China (No. 51576076 and No. 51711540031), Natural Science Foundation of Hubei Province (2017CFA046) and Fundamental Research Funds for the Central Universities (2019kfyRCPY045). An allocation of computer time from the UA Research Computing High Performance Computing (HPC), High Throughput Computing (HTC) at the University of Arizona, the National Supercomputing Center in Tianjin (NSCC-TJ) and China Scientific Computing Grid (ScGrid) are gratefully acknowledged.

\section{References}

1. D. G. Cahill, P. V. Braun, G. Chen, D. R. Clarke, S. Fan, K. E. Goodson, P. Keblinski, W. P. King, G. D. Mahan, A. Majumdar, H. J. Maris, S. R. Phillpot, E. Pop and L. Shi, Appl. Phys. Rev., 2014, 1, 011305.

2. S. Alaie, D. F. Goettler, M. Su, Z. C. Leseman, C. M. Reinke and I. El-Kady, Nat. Commun., 2015, 6, 7228.

3. R. Yanagisawa, J. Maire, A. Ramiere, R. Anufriev and M. Nomura, Appl. Phys. Lett., 2017, 110, 133108

4. E. Dechaumphai and R. Chen, J. Appl. Phys., 2012, 111, 073508-073508.

5. I. El-Kady, R. H. Olsson III, P. E. Hopkins, Z. C. Leseman, D. F. Goettler, B. Kim, C. M. Reinke and M. F. Su, Sandia National Labs, Albuquerque, NM, Report No. SAND2012-0127, 2012.

6. P. E. Hopkins, L. M. Phinney, P. T. Rakich, R. Olsson III and I. El-Kady, Appl. Phys. A, 2011, 103, 575-579.

7. P. E. Hopkins, C. M. Reinke, M. F. Su, R. H. Olsson, E. A. Shaner, Z. C. Leseman, J. R. Serrano, L. M. Phinney and I. El-Kady, Nano Lett., 2010, 11, $107-112$

8. B. Kim, J. Nguyen, P. J. Clews, C. M. Reinke, D. Goettler, Z. C. Leseman, I. El-Kady and R. Olsson, Micro Electro Mechanical Systems (MEMS), 2012 IEEE 25th International Conference on, 2012.

9. M. Maldovan, Nature, 2013, 503, 209.

10. A. M. Marconnet, M. Asheghi and K. E. Goodson, J. Heat Transfer., 2013, 135, 061601

11. M. Nomura, J. Nakagawa, K. Sawano, J. Maire and S. Volz, Appl. Phys. Lett., 2016, 109, 173104

12. M. Verdier, R. Anufriev, A. Ramiere, K. Termentzidis and D. Lacroix, Phys. Rev. B, 2017, 95, 205438.

13. M. R. Wagner, B. Graczykowski, J. S. Reparaz, A. El Sachat, M. Sledzinska, F. Alzina and C. M. Sotomayor Torres, Nano Lett., 2016, 16, 5661-5668.

14. J. K. Yu, S. Mitrovic, D. Tham, J. Varghese and J. R. Heath, Nat. Nanotechnol., 2010, 5, 718-721.

15. Y. Liao, T. Shiga, M. Kashiwagi and J. Shiomi, Phys. Rev. B, 2018, 98 , 134307.

16. M. Maldovan, Phys. Rev. Lett., 2013, 110, 025902.

17. M. Maldovan, Nat. Mater., 2015, 14, 667-674.

18. N. Zen, T. A. Puurtinen, T. J. Isotalo, S. Chaudhuri and I. J. Maasilta, Nat.
Commun., 2014, 5, 3435 .

19. H. Karamitaheri, M. Pourfath, R. Faez and H. Kosina, J. Appl. Phys., 2011, 110, 054506

20. A. Sgouros, M. Sigalas, G. Kalosakas, K. Papagelis and N. Papanicolaou, $J$. Appl. Phys., 2012, 112, 094307.

21. T. G. Pedersen, C. Flindt, J. Pedersen, N. A. Mortensen, A.-P. Jauho and K. Pedersen, Phys. Rev. Lett., 2008, 100, 136804.

22. P. Hariharan, Rep. Prog. Phys., 1991, 54, 339-390.

23. J. Lee, presented in part at the IEEE 1970 IEEE International Conference on Engineering in the Ocean Environment - Digest of Technical Papers, 1970.

24. M. Makarova, J. Vuckovic, H. Sanda and Y. Nishi, 2006.

25. Y. Gong, M. Makarova, S. Yerci, R. Li, M. J. Stevens, B. Baek, S. W. Nam, R. H. Hadfield, S. N. Dorenbos and V. Zwiller, Opt. Express, 2010, 18, $2601-$ 2612.

26. J. Garg and G. Chen, Phys. Rev. B, 2013, 87, 140302.

27. J. Ravichandran, A. K. Yadav, R. Cheaito, P. B. Rossen, A. Soukiassian, S. J. Suresha, J. C. Duda, B. M. Foley, C. H. Lee, Y. Zhu, A. W. Lichtenberger, J. E. Moore, D. A. Muller, D. G. Schlom, P. E. Hopkins, A. Majumdar, R. Ramesh and M. A. Zurbuchen, Nat. Mater., 2014, 13, 168-172.

28. K. Esfarjani, G. Chen and H. T. Stokes, Phys. Rev. B, 2011, 84, 085204

29. A. Jain, Y. J. Yu and A. J. McGaughey, Phys. Rev. B, 2013, 87, 195301.

30. B. Yang and G. Chen, Phys. Rev. B, 2003, 67, 195311.

31. J. M. Ziman, Electrons and phonons: the theory of transport phenomena in solids, Oxford University Press, 2001.

32. Z. M. Zhang, Nano/microscale heat transfer, McGraw-Hill New York, 2007.

33. J. Tang, H.-T. Wang, D. H. Lee, M. Fardy, Z. Huo, T. P. Russell and P. Yang, Nano Lett., 2010, 10, 4279-4283.

34. G. Xie, D. Ding and G. Zhang, Adv. Phys- X, 2018, 3, 1480417

35. J. H. Lee, G. A. Galli and J. C. Grossman, Nano Lett., 2008, 8, 3750-3754.

36. J. H. Lee, J. Grossman, J. Reed and G. Galli, Appl. Phys. Lett., 2007, 91, 223110-223113.

37. L. Yang, N. Yang and B. Li, Nano Lett., 2014, 14, 1734-1738.

38. L. Yang, N. Yang and B. Li, Int. J. Heat Mass Tran., 2016, 99, 102-106.

39. D. Ma, H. Ding, H. Meng, L. Feng, Y. Wu, J. Shiomi and N. Yang, Phys. Rev. B, 2016, 94, 165434.

40. L. Yang, N. Yang and B. Li, Sci. Rep., 2013, 3, 1143.

41. Y. He, D. Donadio, J.-H. Lee, J. C. Grossman and G. Galli, ACS Nano, 2011, 5, 1839-1844.

42. S. Hu, Z. Zhang, P. Jiang, W. Ren, C. Yu, J. Shiomi and J. Chen, Nanoscale, 2019, 11, 11839-11846.

43. Q. Hao, G. Chen and M. S. Jeng, J. Appl. Phys., 2009, 106, 114321/114321114310.

44. Q. Hao, Y. Xiao and H. Zhao, Appl. Therm. Eng., 2017, 111, 1409-1416.

45. Q. Hao, Y. Xiao and H. Zhao, J. Appl. Phys., 2016, 120, 065101.

46. Y. Yan, Q. F. Liang, H. Zhao, C. Q. Wu and B. Li, Phys. Lett. A, 2012, 376 2425-2429.

47. J. Lim, H. T. Wang, J. Tang, S. C. Andrews, H. So, J. Lee, D. H. Lee, T. P. Russell and P. Yang, ACS Nano, 2016, 10, 124-132.

48. Q. Hao, D. Xu, H. Zhao, Y. Xiao and F. J. Medina, Sci. Rep., 2018, 8, 9056.

49. J. Lee, W. Lee, G. Wehmeyer, S. Dhuey, D. L. Olynick, S. Cabrini, C. Dames, J. J. Urban and P. Yang, Nat. Commun., 2017, 8, 14054.

50. D. Song and G. Chen, Appl. Phys. Lett., 2004, 84, 687-689.

51. R. Anufriev, J. Maire and M. Nomura, Phys. Rev. B, 2016, 93, 045411.

52. J. Maire, R. Anufriev, R. Yanagisawa, A. Ramiere, S. Volz and M. Nomura, Sci. Adv., 2017, 3, e1700027.

53. M. Nomura, Y. Kage, J. Nakagawa, T. Hori, J. Maire, J. Shiomi, R. Anufriev, D. Moser and O. Paul, Phys. Rev. B, 2015, 91, 205422.

54. R. Anufriev, A. Ramiere, J. Maire and M. Nomura, Nat. Commun., 2017, 8, 15505 .

55. B. Graczykowski, A. El Sachat, J. S. Reparaz, M. Sledzinska, M. R. Wagner, E. Chavez-Angel, Y. Wu, S. Volz, Y. Wu, F. Alzina and C. M. Sotomayor Torres, Nat. Commun., 2017, 8, 415.

56. D. Xu, Q. Wang, X. Wu, J. Zhu, H. Zhao, B. Xiao, X. Wang, X. Wang and Q. Hao, Frontiers in Energy, 2018, 1-10.

57. T. Klitsner and R. Pohl, Phys. Rev. B, 1987, 36, 6551

58. Y. C. Hua and B. Y. Cao, J. Phys. Chem. C, 2017, 121, 5293-5301.

59. C. Huang, X. Zhao, K. Regner and R. Yang, Physica E, 2018, 97, 277-281.

60. A. Eucken, Ceramic Abstracts, 1932, 11, 576; A. Eucken, Ceramic Abstracts, 
1933, 12, 231

61. Z. Hashin and S. Shtrikman, J. Appl. Phys., 1962, 33, 3125-3131.

62. C. W. Nan, R. Birringer, D. R. Clarke and H. Gleiter, J. Appl. Phys., 1997, 81, 6692-6699.

63. K. D. Parrish, J. R. Abel, A. Jain, J. A. Malen and A. J. McGaughey, J. Appl. Phys., 2017, 122, 125101.

64. A. J. McGaughey and A. Jain, Appl. Phys. Lett., 2012, 100, 061911.

65. A. M. Marconnet, T. Kodama, M. Asheghi and K. E. Goodson, Nanosc. Microsc. Therm., 2012, 16, 199-219.

66. G. Romano and J. C. Grossman, J. Heat Transfer., 2015, 137, 071302.

67. G. Romano and A. M. Kolpak, Appl. Phys. Lett., 2017, 110, 093104.

68. Q. Hao, H. Zhao and D. Xu, J. Appl. Phys., 2017, 121, 094308.

69. H. Casimir, Physica, 1938, 5, 495-500.

70. D. Xu, S. Tang, X. Du and Q. Hao, Carbon, 2019, 144, 601-607.

71. Z. Yu, L. Ferrer-Argemi and J. Lee, J. Appl. Phys., 2017, 122, 244305.

72. M. Nomura, J. Shiomi, T. Shiga and R. Anufriev, Jpn J. Appl. Phys., 2018, 57, 080101

73. H. C. Hottel, WH McAdams. Heat Transmission, 1954.

74. D. Lacroix, K. Joulain and D. Lemonnier, Phys. Rev. B, 2005, 72, 064305.

75. P. E. Hopkins, P. T. Rakich, R. H. Olsson, I. F. El-Kady and L. M. Phinney, Appl. Phys. Lett., 2009, 95, 161902.

76. P. E. Hopkins, L. M. Phinney, P. T. Rakich, R. H. Olsson and I. El-Kady, Appl. Phys. A, 2011, 103, 575-579.

77. A. Minnich and G. Chen, Appl. Phys. Lett., 2007, 91, 073105-073103.

78. G. Chen, Nanoscale Energy Transport and Conversion: A Parallel Treatment of Electrons, Molecules, Phonons, and Photons, Oxford University Press, New York, 2005.

79. H. Machrafi and G. Lebon, Physics Letters A, 2015, 379, 968-973.

80. M. S. Jeng, R. Yang, D. Song and G. Chen, J. Heat Transfer, 2008, 130, 042410-042410-042411.

81. C. Shao, Q. Rong, M. Hu and H. Bao, J. Appl. Phys., 2017, 122, 155104.

82. C. Shao, Q. Rong, N. Li and H. Bao, Phys. Rev. B, 2018, 98, 155418.

83. N. K. Ravichandran, H. Zhang and A. J. Minnich, Phys. Rev. X, 2018, 8, 041004.

84. Q. Hao, Y. Xiao and Q. Chen, arXiv preprint arXiv:1904.08956, 2019

85. Q. Hao, Y. Xiao and Q. Y. Chen, Materials Today Physics, 2019, 10, 100126.

86. Z. Wang, J. E. Alaniz, W. Jang, J. E. Garay and C. Dames, Nano Lett., 2011, 11, 2206-2213

87. M. Kashiwagi, Y. Sudo, T. Shiga and J. Shiomi, Phys. Rev. Appl., 2018, 10, 044018.

88. M. S. Jeng, R. Yang, D. Song and G. Chen, J. Heat Transfer, 2008, 130, 042410.

89. H. Hagino, Y. Kawahara, A. Goto and K. Miyazaki, IOP Conference Series: Materials Science and Engineering, 2012.

90. Y. Hu, L. Zeng, A. J. Minnich, M. S. Dresselhaus and G. Chen, Nat. Nanotechnol., 2015, 10, 701-706.

91. Y. He, D. Donadio and G. Galli, Nano Lett., 2011, 11, 3608-3611.

92. N. K. Ravichandran and A. J. Minnich, Phys.Rev. B, 2014, 89, 205432.

93. R. Anufriev, R. Yanagisawa and M. Nomura, Nanoscale, 2017, 9, 1508315088.

94. A. George, R. Yanagisawa, R. Anufriev, J. He, N. Yoshie, N. Tsujii, Q. Guo, T. Mori, S. Volz and M. Nomura, ACS Appl. Mater. Int., 2019, 11, 1202712031.

95. B. Graczykowski, M. Sledzinska, F. Alzina, J. Gomis-Bresco, J. S. Reparaz, M. R. Wagner and C. M. Sotomayor Torres, Phys. Rev. B, 2015, 91, 075414.

96. A. Iskandar, A. Gwiazda, Y. Huang, M. Kazan, A. Bruyant, M. Tabbal and G. Lerondel, J. Appl. Phys., 2016, 120, 095106.

97. N. W. Ashcroft and N. D. Mermin, holt, rinehart and winston, New York and London, Editon edn., 1976.

98. Q. Hao, D. Xu and H. Zhao, 2015 MRS Spring Meeting \& Exhibit, San Francisco, California, 2015

99. L. Lu, W. Yi and D. Zhang, Rev. Sci. Instrum., 2001, 72, 2996-3003.

100. D. Xu, R. Hanus, Y. Xiao, S. Wang, G. Snyder and Q. Hao, Mater. Today Phys., 2018, 6, 53-59.

101. C. Jeong, S. Datta and M. Lundstrom, J. Appl. Phys., 2012, 111, 093708.

102. M. Kim, N. S. Safron, E. Han, M. S. Arnold and P. Gopalan, Nano Lett., 2010, 10, 1125-1131.

103. A. Sinitskii and J. M. Tour, J. Am. Chem. Soc., 2010, 132, 14730-14732.
104. T. G. Pedersen, C. Flindt, J. Pedersen, A. P. Jauho, N. A. Mortensen and K. Pedersen, Phys. Rev. B, 2008, 77, 245431.

105. J. G. Pedersen, T. Gunst, T. Markussen and T. G. Pedersen, Phys. Rev. B, $2012,86,245410$.

106. T. Gunst, T. Markussen, A. P. Jauho and M. Brandbyge, Phys. Rev. B, 2011, 84, 155449 .

107. Ç. Ö. Girit, J. C. Meyer, R. Erni, M. D. Rossell, C. Kisielowski, L. Yang, C.-H. Park, M. Crommie, M. L. Cohen and S. G. Louie, Science, 2009, 323, 1705-1708.

108. J. H. Seol, I. Jo, A. L. Moore, L. Lindsay, Z. H. Aitken, M. T. Pettes, X. Li, Z. Yao, R. Huang and D. Broido, Science, 2010, 328, 213-216.

109. J. Oh, H. Yoo, J. Choi, J. Y. Kim, D. S. Lee, M. J. Kim, J. C. Lee, W. N. Kim, J. C. Grossman and J. H. Park, Nano Energy, 2017, 35, 26-35.

110. H. Wang, S. Hu, K. Takahashi, X. Zhang, H. Takamatsu and J. Chen, Nat Commun., 2017, 8, 15843.

111. H. Wang, K. Kurata, T. Fukunaga, H. Takamatsu, X. Zhang, T. Ikuta, K Takahashi, T. Nishiyama, H. Ago and Y. Takata, Sci. Rep., 2016, 6, 21823.

112. L. A. Giannuzzi and F. A. Stevie, Micron, 1999, 30, 197-204.

113. V. Iberi, I. Vlassiouk, X. G. Zhang, B. Matola, A. Linn, D. C. Joy and A. J. Rondinone, Sci. Rep., 2015, 5, 11952-11952.

114. M. D. Fischbein and M. Drndić, Appl. Phys. Lett., 2008, 93, 113107.

115. A. Sandner, T. Preis, C. Schell, P. Giudici, K. Watanabe, T. Taniguchi, D. Weiss and J. Eroms, Nano lett., 2015, 15, 8402-8406.

116. R. Yagi, R. Sakakibara, R. Ebisuoka, J. Onishi, K. Watanabe, T. Taniguchi and Y. Iye, Phys. Rev. B, 2015, 92, 195406.

117. Q. Hao, D. Xu, N. Lu and H. Zhao, Phys. Rev. B, 2016, 93, 205206.

118. C. Bera, M. Soulier, C. Navone, G. Roux, J. Simon, S. Volz and N. Mingo, J. Appl. Phys., 2010, 108, 124306.

119. M. F. Modest, Radiative Heat Transfer, Academic press, 2013.

120. J. Bai, X. Zhong, S. Jiang, Y. Huang and X. Duan, Nat. Nanotechnol., 2010, 5, 190-194.

121. J. S. Reparaz, E. Chavez-Angel, M. R. Wagner, B. Graczykowski, J. GomisBresco, F. Alzina and C. M. S. Torres, Rev. Sci. Instrum., 2014, 85, 034901.

122. A. A. Balandin, S. Ghosh, W. Bao, I. Calizo, D. Teweldebrhan, F. Miao and C. N. Lau, Nano Lett., 2008, 8, 902-907.

123. A. K. Vallabhaneni, D. Singh, H. Bao, J. Murthy and X. Ruan, Phys. Rev. B, 2016, 93, 125432

124. L. Yang, J. Chen, N. Yang and B. Li, Int. J. Heat Mass Tran., 2015, 91 , $428-432$.

125. C. Moreno, M. Vilas-Varela, B. Kretz, A. Garcia-Lekue, M. V. Costache, M. Paradinas, M. Panighel, G. Ceballos, S. O. Valenzuela and D. Peña, Science, 2018, 360, 199-203.

126. J. Mahmood, E. K. Lee, M. Jung, D. Shin, I. Y. Jeon, S. M. Jung, H. J. Choi, J. M. Seo, S. Y. Bae and S. D. Sohn, Nat. Commun., 2015, 6, 6486.

127. J. Mahmood, E. K. Lee, M. Jung, D. Shin, H. J. Choi, J. M. Seo, S. M. Jung, D. Kim, F. Li and M. S. Lah, P. Nat. A. Sci., 2016, 113, 7414-7419.

128. D. Nika, A. Cocemasov, C. Isacova, A. Balandin, V. Fomin and O. Schmidt, Phys. Rev. B, 2012, 85, 205439.

129. B. L. Davis and M. I. Hussein, Phys. Rev. Lett., 2014, 112, 055505.

130. S. Xiong, K. Sääskilahti, Y. A. Kosevich, H. Han, D. Donadio and S. Volz, Phys. Rev. Lett., 2016, 117, 025503.

131. R. Anufriev and M. Nomura, Phys. Rev. B, 2017, 95, 155432.

132. B. Li, K. T. Tan and J. Christensen, Physical Review B, 2017, 95, 144305.

133. H. Honarvar and M. I. Hussein, Phys. Rev. B, 2016, 93, 081412.

134. R. Anufriev and M. Nomura, Sci. Technol. Adv. Mat., 2018, 19, 863-870.

135. H. Honarvar, L. Yang and M. I. Hussein, Appl. Phys. Lett., 2016, 108, 263101.

136. D. Ma, X. Wan and N. Yang, Phys. Rev. B, 2018, 98, 245420.

137. J. Chen, G. Zhang and B. Li, J. Chem. Phys., 2011, 135, 104508.

138. M. Hu, K. P. Giapis, J. V. Goicochea, X. Zhang and D. Poulikakos, Nano Lett., 2011, 11, 618-623.

139. J. Chen, G. Zhang and B. Li, Nano Lett., 2012, 12, 2826-2832.

140. Z. Zhang, S. Hu, T. Nakayama, J. Chen and B. Li, Carbon, 2018, 139, 289298.

141. T. Tadano, Y. Gohda and S. Tsuneyuki, Phys. Rev. Lett., 2015, 114, 095501.

142. S. Hu, M. An, N. Yang and B. Li, Nanotechnology, 2016, 27, 265702.

143. S. Hu, M. An, N. Yang and B. Li, Small, 2017, 13, 1602726-n/a.

144. C. Dames, J. Heat Transfer, 2009, 131, 061301. 
145. H. Wang, K. Kurata, T. Fukunaga, H. Takamatsu, X. Zhang, T. Ikuta, K Takahashi, T. Nishiyama, H. Ago and Y. Takata, Sci. Rep., 2016, 6, 21823.

146. K. Konthasinghe, J. Walker, M. Peiris, C. K. Shih, Y. Yu, M. F. Li, J. F. He, L. J. Wang, H. Q. Ni, Z. C. Niu and A. Muller, Phys. Rev. B, 2012, 85, 235315 .

147. C. Rauch, G. Strasser, K. Unterrainer, W. Boxleitner, E. Gornik and A. Wacker, Phys. Rev. Lett., 1998, 81, 3495

148. D. Ma, A. Arora, S. Deng, G. Xie, J. Shiomi and N. Yang, Mater. Today Phys., 2019, 8, 56-61.

149. Y. Wang, H. Huang and X. Ruan, Phys. Rev. B, 2014, 90, 165406.

150. S. Hu, Z. Zhang, P. Jiang, J. Chen, S. Volz, M. Nomura and B. Li, J. Phys. Chem. Lett., 2018, 9, 3959-3968.

151. M. An, Q. Song, X. Yu, H. Meng, D. Ma, R. Li, Z. Jin, B. Huang and N. Yang, Nano Lett., 2017, 17, 5805-5810.

152. M. V. Simkin and G. D. Mahan, Phys. Rev. Lett., 2000, 84, 927.

153. Q. Li and W. Ye, Int. J. Heat Mass Tran., 2017, 107, 534-543.

154. M. N. Luckyanova, J. Garg, K. Esfarjani, A. Jandl, M. T. Bulsara, A. J. Schmidt, A. J. Minnich, S. Chen, M. S. Dresselhaus, Z. Ren, E. A. Fitzgerald and G. Chen, Science, 2012, 338, 936-939.

155. B. Latour, S. Volz and Y. Chalopin, Phys. Rev. B, 2014, 90, 014307.

156. X. Xu, L. F. Pereira, Y. Wang, J. Wu, K. Zhang, X. Zhao, S. Bae, C. T. Bui, R. Xie and J. T. Thong, Nat. Commun., 2014, 5, 3689.

157. K. Choi, M. Arita and Y. Arakawa, J. Cryst. Growth, 2012, 357, 58-61.

158. G. Romano and J. C. Grossman, Appl. Phys. Lett., 2014, 105, 033116.
159. W. Park, J. Sohn, G. Romano, T. Kodama, A. Sood, J. S. Katz, B. S. Y. Kim, H. So, E. C. Ahn, M. Asheghi, A. M. Kolpak and K. E. Goodson, Nanoscale, 2018, 10, 11117-11122.

160. J. Miller, W. Jang and C. Dames, ASME 2009 Heat Transfer Summer Conference collocated with the InterPACK09 and 3rd Energy Sustainability Conferences, 2009.

161. M. Schmotz, J. Maier, E. Scheer and P. Leiderer, New J. Phys., 2011, 13, 113027.

162. S. Gluchko, R. Anufriev, R. Yanagisawa, S. Volz and M. Nomura, Appl. Phys. Lett., 2019, 114, 023102.

163. Y. Kage, H. Hagino, R. Yanagisawa, J. Maire, K. Miyazaki and M. Nomura, Jpn. J. Appl. Phys., 2016, 55, 085201.

164. A. A. Maznev, A. G. Every and O. B. Wright, Wave Motion, 2013, 50, 776784.

165. M. N. Luckyanova, J. Mendoza, H. Lu, B. Song, S. Huang, J. Zhou, M. Li, Y. Dong, H. Zhou, J. Garlow, L. Wu, B. J. Kirby, A. J. Grutter, A. A Puretzky, Y. Zhu, M. S. Dresselhaus, A. Gossard and G. Chen, Sci. Adv., 2018, 4, eaat9460.

166. S. Ju and J. Shiomi, Nanosc. Microsc. Therm., 2019, 23, 157-172.

167. M. Ohnishi and J. Shiomi, APL Mater., 2019, 7, 013102.

168. J. Maire, R. Anufriev, T. Hori, J. Shiomi, S. Volz and M. Nomura, Sci. Rep$U K, 2018,8,4452$

Publisher's Note Engineered Science Publisher remains neutral with regard to jurisdictional claims in published maps and institutional affiliations. 